\title{
Pour une nouvelle approche du prélèvement
} seigneurial

L'exemple du pays de Retz à la fin du Moyen Âge

\section{Brice Rabot}

\section{OpenEdition}

\section{Journals}

Édition électronique

URL : http://journals.openedition.org/abpo/3208

DOI : $10.4000 / a b p o .3208$

ISBN : : 978-2-7535-5040-7

ISSN : 2108-6443

Éditeur

Presses universitaires de Rennes

\section{Édition imprimée}

Date de publication : 22 avril 2016

Pagination : 85-112

ISBN : 978-2-7535-5038-4

ISSN : 0399-0826

\section{Référence électronique}

Brice Rabot, «Pour une nouvelle approche du prélèvement seigneurial », Annales de Bretagne et des Pays de l'Ouest [En ligne], 123-1 | 2016, mis en ligne le 22 avril 2018, consulté le 02 mai 2019. URL: http://journals.openedition.org/abpo/3208; DOI : 10.4000/abpo.3208 


\title{
Pour une nouvelle approche du prélèvement seigneurial
}

\section{L'exemple du pays de Retz à la fin du Moyen Âge}

\author{
Brice RABOT \\ Docteur en histoire médiévale - université de Nantes \\ Chercheur associé au Centre de Recherches en Histoire Internationale et Atlantique
}

(CRHIA)

Le prélèvement seigneurial, qui regroupe les droits et les redevances perçus par les seigneurs ou les détenteurs de droits et de pouvoirs sur d'autres hommes ainsi que sur la terre, constitue, selon l'expression de Monique Bourin, un véritable " nœud de l'histoire médiévale ${ }^{1}$ ". L'historiographie récente invite à l'envisager sous de nouveaux regards en croisant les approches et les démarches pour en étudier les modalités concrètes, le calendrier des levées, le personnel chargé des collectes ou encore les relations d'homme à homme mises au jour par les gestes et les rituels de la perception. Pour être menée à bien, une telle étude doit s'inscrire dans un cadre restreint et cohérent. En Bretagne méridionale, le pays de Retz constitue à cet égard un exemple approprié. Dominé par l'une des plus importantes baronnies bretonnes, le pays de Retz englobe la quasitotalité de la rive sud de la Loire ${ }^{2}$, aux confins du Poitou. Les relations des barons ${ }^{3}$ avec leurs vassaux contribuent également à assurer l'unité de ce territoire.

1. Bourin, Monique, Martínez SoPena, Pascual (dir.), Pour une anthropologie du prélèvement seigneurial dans les campagnes médiévales (XI -XIV siècles), colloque tenu à Medina del Campo du 31 mai au 3 juin 2000, Paris, Publications de la Sorbonne, 2004, p. 11.

2. Le pays de Retz se décompose en réalité en deux grands ensembles avec des enjeux différents pour l'étude du prélèvement. La frange littorale, avec l'exploitation du sel, présente des caractéristiques qui dépassent notre propos et a déjà été l'objet de l'attention des historiens. Nous renvoyons à cet égard à la présentation de HOCQUET, Jean-Claude, SARRAZIN, Jean-Luc (dir.), Le sel de la baie : histoire, archéologie, ethnologie des sels atlantiques, Actes du colloque "Le sel de la Baie et ses concurrents ", Rennes, PUR, 2006. Seul l'arrière-pays sera pris en considération dans cette contribution.

3. Aux XIV et Xve siècles, le terme " barons " n'apparaît pas dans les sources du pays de Retz. Le terme " baronnie " en revanche est davantage usité. Pour désigner le détenteur 
Sans être exceptionnelle, la documentation disponible permet d'envisager la question du prélèvement sous différents angles. Le cartulaire des sires de Retz ${ }^{4}$ éclaire d'abord les relations des sires de Retz avec leurs vassaux, mais aussi avec les ducs de Bretagne lors des opérations de rachat. Les seigneuries de La Blanchardais et des Huguetières, vassales de Retz, avec leurs larges séries comptables du $\mathrm{Xv}^{\mathrm{e}}$ siècle et de nombreux aveux, permettent ensuite d'analyser les modalités d'organisation des levées ${ }^{5}$. Les fonds de l'abbaye cistercienne de Buzay ${ }^{6}$ jettent quelques éclairages sur les modalités de règlement des conflits, notamment pour les arriérés de rentes ${ }^{7}$. Les archives des autres seigneuries, davantage clairsemées, présentent enfin l'assise foncière sur laquelle pèsent les principales rentes ${ }^{8}$.

Après avoir présenté les principales sources disponibles et les perspectives qu'elles ouvrent, l'étude esquissera un tableau des formes du prélèvement et s'achèvera par une réflexion sur les modalités de perception des redevances.

\section{Pour un nouvel examen des sources}

\section{Les aveux}

Les aveux figurent parmi les sources les plus abondantes pour aborder le prélèvement, quel que soit le type de seigneurie. Deux grandes catégories peuvent être distinguées. Les aveux pour rachat, rassemblés dans la série $B$ des Archives départementales de Loire-Atlantique, sont les plus nombreux. Adressés au duc de Bretagne, ils sont remis en réalité aux officiers de la Chambre des comptes par les seigneurs ou leurs représentants légaux en cas de minorité. Établis au moment des successions, ces actes répondent

de la baronnie de Retz, les rédacteurs de la fin du Moyen Âge emploient les mots « sire " ou " seigneur".

4. Ce cartulaire rassemble différents actes (aveux, contestations, décisions de justice, etc.) établis ou reçus par les sires de Retz entre le $\mathrm{XIII}^{\mathrm{e}}$ et le $\mathrm{XV}^{\mathrm{e}}$ siècle (Cartulaire des sires de Rays (1160-1449), publié par Blanchard, René, Archives historiques du Poitou, tome 30, Poitiers, 1899, p. 118-120, 124-125, 128-129, 132-133, 147, 228-236, 240-245. Disponible sur le site en ligne Gallica).

5. Arch. dép. de Loire-Atlantique, 1 E 221 à 223 (La Blanchardais, de 1430 à 1513); E 500 à 502 (Huguetières, de 1405 à 1493).

6. Cette abbaye nous a légué un important chartrier (SARRAzIN, Jean-Luc, Recueil et catalogue des actes de l'abbaye cistercienne de Buzay en Pays de Rais (1135-1474), thèse de troisième cycle (dactyl.), 4 tomes, Université de Nantes, 1977). Organisée autour de Rouans, au cœur du pays de Retz, l'abbaye de Buzay dispose aussi de tenures au nord du fleuve, à Cordemais, à Saint-Étienne-de-Montluc ou à Savenay avec les donations et les autres fondations effectuées au cours du temps par les fidèles (DuFIEF, André, Les Cisterciens en Bretagne (XII'-XII' siècles), Rennes, PUR, 1997, p. 75-78, 88-89, 118-119, 137 139, 168-176).

7. Arch. dép. de Loire-Atlantique, E 80, H 25, H 34, H 39, H 55.

8. Par exemple, pour la châtellenie de La Bénâte : Arch. dép. de Loire-Atlantique, E 489; pour la châtellenie de Machecoul : Arch. dép. de Loire-Atlantique, E 522. 
d'abord à un objectif fiscal en présentant les revenus totaux du seigneur ${ }^{9}$. Ils permettent ensuite de saisir la nature des patrimoines en citant des listes plus ou moins longues de biens et d'assujettis. Les terres et les maisons concédées ${ }^{10}$, quelle que soit la seigneurie, occupent la majeure partie du dispositif, en raison des nombreux droits qui pèsent sur elles; les terres de la réserve, exploitées par les métayers, ne sont, à l'inverse, que très rarement mentionnées ${ }^{11}$. Les aveux pour rachat n'apportent, de manière générale, aucune indication sur l'exploitation de la réserve seigneuriale.

Les aveux pour rachat revêtent aussi une dimension ostentatoire : ils offrent l'occasion aux seigneurs de rappeler l'étendue de leurs droits et prérogatives en les énumérant plus ou moins précisément ${ }^{12}$. Tous insistent régulièrement sur l'obéissance due par les tenanciers ${ }^{13}$. Tous prennent soin de recenser l'ensemble des droits dus pour éviter toute contestation ultérieure et pour pouvoir effectuer correctement les levées.

Les aveux rendus par les vassaux ou les tenanciers, en dehors des rachats ou à cette occasion, présentent d'autres caractéristiques ${ }^{14}$. Établis à la demande du seigneur, ils commencent toujours par préciser la nature des relations entretenues avec le seigneur foncier ${ }^{15}$. La comparaison des

9. Le montant du rachat varie selon les ressources seigneuriales. Il correspond en général à une année de revenus (RABOT, Brice, Les structures seigneuriales en Bretagne méridionale (comté de Nantes et pays vannetais) à la fin du Moyen Âge (XIVe-début XVe siècle), thèse de doctorat (dactyl.), 2 tomes, université de Nantes, 2014, p. 93).

10. Ibidem, p. 190-192. Par exemple : « Item quinze sols deux chappons de rente que me devent les hoirs Jehan Dain sur leur censive et appartenances ", extrait d'un aveu rendu le 12 décembre 1451 par le seigneur du Chaffaut au duc de Bretagne pour des tenures situées à Saint-Colomban (Arch. dép. de Loire-Atlantique, E 150). Les censives ne sont pas toujours clairement mentionnées : "C'est assavoir la moitié du herbrégement, domaine, mesons, courtilz, rues, yssues et appartenances du Boais Corbeau, comme il se poursuist, tant terres, boays, tailleys et de revenues et contenant saexante boixellées de terre ou environ ", extrait de l'aveu rendu par René de Quersy devant la cour du Pellerin le 14 mars 1483 (n. st.) (Arch. dép. de Loire-Atlantique, B 1829).

11. Les aveux ne contiennent aucune information quant au nombre ou à l'implantation exacte des métairies : « hommes, homaiges, rachaz, soulz rachatz d'espaves, galoiz, droit de quictances, de désérence et succession de bastars, de ventes et des mestaeries, terrages [...] et autres héritages, droiz héritelz et choses héritelles quexlconques estans des appartenances et appendances dicelle terre et seigneurie de La Bénaste ", extrait de l'aveu pour rachat rendu le 17 août 1462 par la dame de La Suze (Arch. dép. de Loire-Atlantique, B 1816).

12. Par exemple : " estoit tenu de faire à cause et pour raison des baronnies, chasteaux, chastellenies, terres et seigneuries de Rays, Saint-Estienne-de-Malemort, Bourgneuf, La Bénaste, et autres terres et seigneuries que tenoit et possidoit ledit feu seigneur de Rays au temps de son décès ", extrait de l'aveu rendu le 2 octobre 1443 (Cartulaire des sires de Rays..., op. cit., pièce LXxXvI, p. 244).

13. Par exemple : " obéissance comme homme estaigier et doit à son seigneur et selont que le fié et les tenues le requièrent et qu'il doit tenir de nostre dit souverain seigneur et de ses officiers ", extrait de l'aveu rendu le 14 mars 1483 (n. st.) (Arch. dép. de LoireAtlantique, B 1829).

14. Ces aveux sont pour l'essentiel regroupés dans la sous-série $1 \mathrm{E}$ des Archives départementales de Loire-Atlantique, intitulée " Titres féodaux ".

15. Par exemple, les moines de Blanche-Couronne, implantés à Savenay, rendent régulièrement hommage aux sires de Retz pour leurs possessions dans la châtellenie de Pornic, 
aveux conduit à se poser d'autres questions. Le prélèvement est-il le même dans les grandes structures que dans les petites? Pèse-t-il plus lourd selon que le seigneur dispose de droits étendus ou n'est-il pas, au contraire, plus fort pour les tenanciers des petites structures, aux ressources limitées? La mise en relation des sommes versées pour les cens, par exemple, ne montre pas de réelle différence ${ }^{16}$. Les redevances se diversifient en revanche très nettement au fur et à mesure que l'on s'élève dans la hiérarchie vassalique, jusqu'à atteindre les châtellenies ${ }^{17}$. L'exercice de la justice par exemple donne lieu à la perception de taxes spécifiques ${ }^{18}$. La nature des liens qui relient les vassaux aux sires de Retz, les relations développées avec d'autres seigneurs, extérieurs ou non au pays de Retz, expliquent, pour partie, ces différences.

Les aveux présentés par les tenanciers, en leur nom ou au nom de leur famille, établissent des listes plus restreintes de rentes et de droits sur les biens concédés. Ces aveux ne se contentent pas de préciser les montants; ils laissent voir également les rapports entretenus entre seigneurs et paysans à travers le prélèvement. Les tenanciers reconnaissent toujours, à la fin de chaque acte, devoir obéissance au seigneur foncier ${ }^{19}$. Certains se

à La Plaine-sur-Mer : " par union, fusmes à gré confessans que nous tenions et tenons de vous nostre puissant et noble, en voustre terrouer de Rays, en la parroisse de NostreDame de La Plenne, en la chastelenie de Pornic, c'est assavoir : toutes et chascunes les rentes et revenues que nous avons et levons et avons acoustumé à lever et recevoir en ladicte parroisse, en ladicte chastellenie, tant en rentes par deniers comment que ilz soient dictes et appellés, fromment de rente, terrages, cornages, reserff de bestes non taillées ou cornaige, selon usement et gouvernement de païs, desmes de blez, de vins, de lainnes, d'aignaux novaux, juridicions, obboïssances ", extrait de l'aveu rendu le 22 janvier 1403 (n. st.) par l'abbé de Blanche-Couronne au sire de Retz (Cartulaire des sires de Rays..., op. cit., pièce CCLXXIX, p. 396-397).

16. Les cens ne dépassent guère, par tenancier et par an, les quelques sous. Comme, par exemple, pour la châtellenie de Machecoul (Arch. dép. de Loire-Atlantique, E 522). Il en est de même pour la châtellenie des Huguetières : SADDIER, Évelyne, Une seigneurie rurale du pays nantais au $X^{e}$ siècle : les Huguetières à travers les comptes du receveur, mémoire de DEs (dactyl.), Centre universitaire de Savoie, Chambéry, 1975, p. 41-43.

17. "Sachent touz que je, Bretran de Dinan, seigneur des Uguetières, confesse estre homme féal de noble et puissant seigneur monseigneur de Rays, et de lui confesse et avoue tenir noblement, à fay et à rachat, quant le cas y avienent, savoir est : ma terre et chastelenie des Uguetières, comme elle se poursiet o toutes et chascune ses appandences et deppendances, et tant en domaines, hommes, homaiges, juridicions, seigneuries et obbéissances, bouais, forestz, esves, molins, rentes par blez et deniers, gélines, biangs, corvées, espaves, galoaiz et aultres droiz hériteulx et seigneurieulx quelxconques; et généralement tout ce que j'ay et à moy appartient de héritaige, rentes et revenues et droiz hériteulx quelxconques ou duché de Bretaigne entre la rivière de Loire et le païs de Poictou, tant en Marche que autrement, sans riens en excepter ne retenir ", vidimus de l'aveu rendu le 18 septembre 1428 par Bretran de Dinan, seigneur des Huguetières, au seigneur de Retz (Cartulaire des sires de Rays..., op. cit., pièce cCXLI, p. 307-308).

18. Les revenus tirés de l'exercice de la justice s'élèvent, en moyenne, à une centaine de livres par an pour la châtellenie des Huguetières, soit un dixième environ des recettes totales (SADDIER, Évelyne, Une seigneurie rurale..., op. cit., p. 85).

19. «Et en oultre fut et est ledit Chace confessant devoir audit Guillaume Blanchart, à cause de sesdiz héritaiges, ferme droit et obéissance, comme homme de foy doit à son 
déclarent même hommes " estagiers $^{20}$ ". Ces rappels, ajoutés par les rédacteurs, sont loin d'être anodins : ils traduisent la sensibilité des seigneurs aux signes de reconnaissance de leur autorité, particulièrement au moment du paiement, avec les gestes et les rituels de la perception.

Certaines questions restent néanmoins en suspens. La taille des exploitations, par exemple, demeure mal connue. Des fragments laissent entrevoir des parcelles modestes, mais nos connaissances sont trop limitées pour être généralisées ${ }^{21}$. Le rendement des exploitations ainsi que la part totale des revenus paysans consacrée au prélèvement nous échappent également. Ces données ne sont pas nécessaires à l'établissement des aveux.

\section{Les comptes}

Sur le modèle de l'administration ducale dont elles s'inspirent, les comptabilités seigneuriales deviennent, au Xv ${ }^{\mathrm{e}}$ siècle, d'usage courant ${ }^{22}$. Les aléas de la conservation et les destructions opérées au fil des siècles ont conduit à de nombreuses pertes. La châtellenie de Machecoul, avec seulement deux registres transmis ${ }^{23}$, est emblématique à cet égard. D'autres séries, par leur étendue chronologique et leur bonne conservation, offrent de précieuses informations, à l'instar de la seigneurie de La Blanchardais (Vue). Seize registres nous sont ainsi parvenus pour la période $1429-1513^{24}$. Cet exemple, exceptionnel pour le pays de Retz, peut être remis en perspective avec les registres de la châtellenie voisine des Huguetières (La Chevrolière) ${ }^{25}$. Confrontées aux aveux, les comptabilités constituent des sources fondamentales en croisant les regards économiques et anthropologiques.

Le premier intérêt des comptabilités est de dresser, à l'issue de chaque exercice comptable, un bilan annuel des redevances perçues et

seigneur et comme les choses le doivent. ", extrait de l'aveu rendu le 21 mai 1416 par Perrot Chace (Arch. dép. de Loire-Atlantique, 1 E 217).

20. «Et par ces présentes, congnoessent et avouent estre homes et subgitz estagiers de noble escuier Gérard Blanchart, seigneur de La Blanchardaye et de La Vaerie, leur seigneur. ", extrait de l'aveu rendu le 8 mars 1478 (n. st.) par la famille Bourreau (Arch. dép. de Loire-Atlantique, 1 E 217).

21. La définition des mesures pose de redoutables problèmes : elles varient très fortement d'une seigneurie à l'autre. Nous ne disposons que de quelques données, anciennes, établies à la fin du XVIII e siècle (DEmolon, Manuel décimal de l'arpenteur, Nantes, 1802). D'une manière générale, en pays de Retz, la boisselée de terre équivaut à 14 ou 15 ares, tandis que l'hommée de vigne représente en moyenne 6 à 7 ares. Les actes de la pratique, en particulier la série H 39, indiquent majoritairement des tenures de deux boisselées.

22. KeRHERVÉ, Jean, L'État breton aux XIV et XV siècles. Les ducs, l'argent et les hommes, Paris, Maloine, 1987, t. 1, p. 411-416.

23. Seuls deux registres, couvrant les années 1462-1463 et 1497-1498, nous sont parvenus (Arch. dép. de Loire-Atlantique, E 522).

24. Les registres de La Blanchardais couvrent en réalité 48 années sur toute cette période (Arch. dép. de Loire-Atlantique, 1 E 221 à 223).

25. Les modalités d'organisation, le poids des redevances dans les recettes totales peuvent ainsi être comparés plus facilement qu'à travers les aveux. 
des dépenses effectuées par le receveur au nom du seigneur. Les progrès accomplis au cours du Xve siècle, matérialisés par des rubriques de mieux en mieux structurées et présentées, permettent de comparer les redevances entre elles (fermes, redevances liées à l'exercice de la justice ou au pouvoir de ban, etc.) mais aussi par rapport aux recettes totales. Les progrès concernent aussi les modalités de perception. L'établissement de bilans partiels, les précisions de plus en plus régulières apportées par les receveurs eux-mêmes, la tutelle des seigneurs ${ }^{26}$ révèlent une attention croissante portée aux outils de gestion ${ }^{27}$.

Les comptabilités permettent ensuite d'appréhender des évolutions passées sous silence par les aveux. Les fermes par exemple ne sont guère connues en dehors des comptes. Elles occupent une place croissante dans les registres du $\mathrm{XV}^{\mathrm{e}}$ siècle ${ }^{28}$, preuve de la réorientation progressive des structures du prélèvement. Les difficultés de la fin du XV $\mathrm{XV}^{\mathrm{e}}$ siècle accélèrent ce processus entamé, d'après les indications disponibles, au tournant des années $1460^{29}$.

Les comptabilités éclairent justement les aléas pesant sur les seigneuries à la fin $\mathrm{du} \mathrm{XV}^{\mathrm{e}}$ siècle. Les épisodes météorologiques violents ${ }^{30}$, parfois désastreux, les désordres, avec la guerre ou la fuite des paysans ${ }^{31}$, les mutations monétaires des années 1480 sont autant de menaces pour l'équilibre

26. La présence de Gérard Blanchart, seigneur de La Blanchardais, lors de certaines enchères, montre le soin porté à l'exploitation de son domaine : " Du prouffit et revenue de la ferme de la terre du pré à la Chevalière, du Grant Faucheis et du Petit Faucheis, affermé par ce receveur, en la présence de monseigneur, à Micheau Borreau, comme plus donnans, l'an mil IIIIC LXI 9 livres. "Arch. dép. de Loire-Atlantique, 1 E 222 (1), f 8 v $^{\circ}$.

27. Les registres dépassent de plus en plus la trentaine de folios, même si tous ne sont pas forcément utilisés. Les cahiers de comptes de La Blanchardais comprennent, par exemple, 40 feuillets en 1486 (Arch. dép. de Loire-Atlantique, 1 E 223 [2]), contre 24 en 1450 (Arch. dép. de Loire-Atlantique, 1 E 221 (3)].

28. En 1462, 18 feuillets sur 29 leur sont consacrés dans les registres des receveurs de La Blanchardais (Arch. dép. de Loire-Atlantique, 1 E 222 [1], f $4 \mathrm{r}^{\circ}-14 \mathrm{v}^{\circ}, 18 \mathrm{r}^{\circ}-20 \mathrm{v}^{\circ}$ ). En 1491, les fermes occupent jusqu'à 10 feuillets sur 15 (Arch. dép. de Loire-Atlantique, 1 E $\left.223[3]), \mathrm{f}^{\circ} 4 \mathrm{r}^{\circ}-3 \mathrm{v}^{\circ}\right)$.

29. Les déficits se multiplient dans les années 1480-1490, ce qui montre la difficulté des seigneurs à faire face aux changements brutaux liés à la conjoncture : RABOT, Brice, Les structures seigneuriales..., op. cit., p. 500.

30. Par exemple : «Et auxi ne compte pas ledit receveur de la ferme de la levée des chomées du Soubsain Deffais, ne de la ferme des chemées du marois qui fut Bidoit, dudit an mil IIIIC XXXVI, pour ce que ils ne furent point venduz pour les esves qui les submergèrent et ne asséchèrent pour celui an pareillement du pré à la dame. " (Arch. dép. de LoireAtlantique, 1 E 221 [2], $\mathrm{f}^{\circ} 4 \mathrm{r}^{\circ}$ ).

31. Comme par exemple en 1513-1514 pour la ferme du moulin de Vue : «Pour ung moys cinq jours de tiers an de la ferme [dudit] molin, affermé audit Jehan Raimbault, le nombre de cinquante boexeaux seille par an, comanczé iceluy moys le dixiesme jour d'aougst l'an $\mathrm{V}^{\mathrm{CC}}$ treze et finit le $14^{\mathrm{e}}$ jour de septembre après ensuyvant, pourtant que ledit Raimbault ne voulist parachever sadicte ferme et icelle relingna et se absenta du pays. Par quoy fut, ledit molin, affermé par cedit receveur à plus grant nombre [de blé] que n'en poiet ledit Raimbault, ainsi que cy après sera descleré. Pour ce, pour ledit moys, qui vault audit pris de cinquante boexeaulx, le nombre de 4 boexeaux 3 quars de boexeau seille. " Arch. dép. de Loire-Atlantique, 1 E 223 (6), $\mathrm{f}^{\circ} 21 \mathrm{r}^{\circ}$. 
du prélèvement. Les annotations des receveurs, les ratures et les rectifications apportées par les auditeurs lors du contrôle des bilans comptables, soulignent à quel point les situations demeurent fragiles ${ }^{32}$ et nécessitent des mises à jour régulières.

\section{Les rentiers et censiers}

Les rentiers ou censiers ${ }^{33}$, présentés sous forme de registres ou de rôles, constituent d'autres instruments indispensables pour les seigneurs et leurs agents. Les fonds d'archives reflètent mal aujourd'hui cette réalité en ne présentant que des actes épars et isolés ${ }^{34}$.

Véritables outils pour la mise à jour des redevances et des redevables, les rentiers sont régulièrement consultés par les receveurs. Les renvois de plus en plus fréquents dans les comptes du Xv $\mathrm{Xv}^{\mathrm{e}}$ siècle en témoignent ${ }^{35}$. Les énumérations de tenanciers et de rentes sont en parallèle plus rares dans les registres. L'insertion de nouvelles listes, établies sur le modèle des rentiers, n'est plus effectuée que lorsque les conditions l'imposent. Les mises à jour ne se substituent pas pour autant aux rentiers : elles ne comportent en effet guère de précisions sur la nature des biens ${ }^{36}$, car les receveurs n'en ont pas besoin. Elles permettent en revanche d'entrevoir les structures familiales paysannes. Les frérèches, régulièrement citées, sont essentielles pour lever le prélèvement ${ }^{37}$; elles renforcent aussi la cohésion

32. Les registres de la décennie 1480 sont à cet égard révélateurs : " Le compte que à noble escuier Gérard Blanchart, seigneur de La Blanchardaie, de La Vayre et de l'Isle Tizon, rend Pierres Bochays, receveur dudit escuier en sa terre et seignorie de Veuz, des receptes et mises par luy faictes, en ce qu'est ladicte recepte, dempuix le terme et feste de Pasques entrant l'an mil IIIIC IIIIXx VII, celuy terme non comprins, et après lequel terme passé y eut mutacion et changement de monnoie et eurent cours les gros à 2 soulz 6 deniers chacun " (Arch. dép. de Loire-Atlantique, 1 E 223 (3) $\mathrm{f}^{\circ} 1 \mathrm{r}^{\circ}$ ). Les auditeurs sont alors obligés de rectifier les sommes inscrites sur les registres, avec un risque d'erreur dans les calculs, d'autant que plusieurs systèmes monétaires coexistent : "Pour lesdictes rentes de l'an mil IIIIC IIIIxx se charge [rayé] deiz se charge de [rayé] et IIIIxx XI par chacun dycelx 6 livres 7 soulz 8 deniers obole est somme 6 livres 7 soulz 8 deniers obole bonne monnoie [rayé et corrigé en : 12 livres 15 soulz 5 deniers bonne] " (ibid., $\mathrm{f}^{\circ} 1 \mathrm{v}^{\circ}$ ).

33. Ils sont parfois confondus avec les minus. Par exemple : "Au terme de la mykaresme l'an mil IIIIC XXIX, selon le minu autreffois lui baillé pour faire la levée desdictes rentes, pour ce 5 soulz 8 deniers. " Arch. dép. de Loire-Atlantique, 1 E 221 (1), f ${ }^{\circ} 1 \mathrm{v}^{\circ}$.

34. Le seul exemple disponible pour le pays de Retz se situe en marge du territoire (Arch. dép. de Loire-Atlantique, B 1853 [Maisdon-sur-Sèvre]).

35 . Au cours du $\mathrm{XV}^{\mathrm{e}}$ siècle, les receveurs remplacent de plus en plus les longues listes de tenanciers et de biens par des bilans partiels, fondés sur les cahiers précédents, sans préciser lesquels ont été mobilisés : «Item, se charge des rentes à deniers certaines deues au terme de la myaougst l'an mil IIIIC LXVI, selon les comptes précédens et rolles rentiers anciens, qui montèrent pour ledit an 6 livres 7 soulz 8 deniers obole. " Arch. dép. de LoireAtlantique, 1 E $222(3), \mathrm{f}^{\circ} 1 \mathrm{v}^{\circ}$.

36. Par exemple, pour La Blanchardais (Arch. dép. de Loire-Atlantique, 1 E 223 [1], $\mathrm{f}^{\circ} 1 \mathrm{r}^{\circ}$ à $11 \mathrm{r}^{\circ}$ ).

37. Par exemple, pour La Blanchardais (Arch. dép. de Loire-Atlantique, 1 E 223 [1], $\left.\mathrm{f}^{\circ} 3 \mathrm{r}^{\circ}, 5 \mathrm{r}^{\circ}, 8 \mathrm{v}^{\circ}, 9 \mathrm{v}^{\circ}, 15 \mathrm{v}^{\circ}\right)$. 
des communautés paysannes en faisant vivre sur les mêmes tenures des ensembles familiaux plus ou moins larges ${ }^{38}$.

La meilleure articulation entre comptes et rentiers ne s'accompagne pas, dans la seconde moitié du $\mathrm{XV}^{\mathrm{e}}$ siècle, d'une hausse des rentes pour les tenanciers. Elle traduit plutôt une réorientation majeure des comptabilités avec la conservation et l'utilisation plus fréquente, par les receveurs, des registres antérieurs pour mieux exiger le prélèvement, à un moment où la conjoncture les oblige justement à être très attentifs.

\section{Les actes de procédure}

Même s'ils ne dévoilent que des vues partielles, les actes de procédure offrent le grand avantage de présenter des cas concrets, qui éclairent sous un autre angle le fonctionnement des seigneuries. Les destructions, les aléas de la conservation ont conduit là encore à de nombreuses pertes ${ }^{39}$, qui empêchent d'étudier aujourd'hui les rouages des justices seigneuriales bretonnes. Les sources ne portent en outre que sur la fin des procédures, avec les condamnations prononcées par les plaids seigneuriaux, et ne concernent qu'un nombre restreint de seigneuries. La bonne conservation du chartrier des moines de Buzay révèle, par exemple, le soin accordé à la gestion du domaine. Aux XIV et $\mathrm{XV}^{\mathrm{e}}$ siècles, les moines sont régulièrement amenés à engager des actions contre les tenanciers qui refusent de payer les dîmes ${ }^{40}$ ou les rentes ${ }^{41}$. Les sommes en jeu demeurent modestes pour celles qui peuvent être saisies, puisqu'elles ne dépassent guère une dizaine

38. Les rentiers n'apportent guère d'informations sur la composition exacte des frérèches : "Perrot Bachelon, Perrot Allain, à cause de sa femme, et leurs consors héritiers de Richart Bachelon, Jehan Bachelon, Guillaume Bachelon, sur les héritaiges dyceulx dont les noms sont cy devant desclerez 3 boexeaux seille. " Arch. dép. de Loire-Atlantique, 1 E $223(1), \mathrm{f}^{\circ} 15 \mathrm{v}^{\circ}$.

39. Les bombardements durant la Seconde Guerre mondiale (1939-1945), particulièrement en 1943, ont ravagé la ville de Nantes et entraîné de lourdes pertes archivistiques comme les chartriers ou les registres d'assises. Voir à ce sujet : THOMAS, Patrick, Nantes, les bombardements (1940-1944), Montreuil-Bellay, CMD, 1996, p. 117.

40. Les actes sont rassemblés sous la cote H 55 des Archives départementales de LoireAtlantique.

41. Par exemple : " Présent en nostre court de Rays, à Pornic, Robin Prodomme, parroessien du bourgc Saincte-Marie, ainxi qu'il disoit, lequel de sa bonne [et] libérale volumpté et sens aucun pour forcement mes à sa requeste a esté et est congnoessant et confessant debvoir et qui doibt de fait à Colin Coguen et Georget Janvier en leurs noms et comme fermiers du priouré du Porteau, ainxin que ledit Robin Proudomme a appoié en nostre présence la somme et numbre de seix soulz monnaie à cause et par rayson des erréaiges de doux soulz de rente que ledit Robin a cognu devoir par an par devant nous audit priouré du Porteau, quelle somme de seix soulz monnaie à cause de l'erréaige desdiz deux soulz de rente restans des troys ans derrains ainxi qu'il a esté a poié en nostre présence ledit Proudomme a voulu promis et s'est obligé, promet et soy oblige rendre poier et bailler esdiz Coguen et Janvier esdiz noms ladicte somme de seix soulz monnaie, ainxi que dit est, dedans quinze jours prochains bien sur l'ipothecque et obligacion de touz et chacun ses biens meubles et immeubles présens et advenir ", extrait de la condamnation prononcée par la cour de Pornic le $1^{\text {er }}$ décembre 1491 (Arch. dép. de Loire-Atlantique, H 10). 
de sous par $\mathrm{an}^{42}$. Le principal enjeu n'est donc pas seulement économique. Les moines engagent des poursuites car le non-paiement des rentes remet en cause l'autorité symbolique liée au prélèvement. Ils ne peuvent dans ces conditions ni tolérer, ni laisser perdurer une telle situation. Chaque condamnation se conclut d'ailleurs par le rappel fait aux tenanciers d'avoir à s'acquitter des redevances à l'avenir ${ }^{43}$, sans qu'il soit fait état d'amendes en cas d'infraction. Ces rappels sont là aussi symboliques ${ }^{44}$.

Les moines de Buzay, comme les autres seigneurs, peuvent recourir à d'autres modes pour régler les conflits. Les médiations, en dehors des actions de justice ou parallèlement à celles-ci, sont en effet attestées ${ }^{45}$. Le règlement du contentieux peut intervenir avant que la justice n'ait à se prononcer, entraînant l'abandon des poursuites. Les tenanciers peuvent aussi faire appel à la solidarité, en premier lieu familiale, pour régler les dettes avant la conclusion de la procédure. Les rentes à assembler en une seule main sont particulièrement propices, en permettant aux paysans de mieux répartir le poids des redevances. De tels accommodements n'ont pas laissé de traces directes dans les archives, car ils n'entravaient pas la perception.

D’après la documentation disponible, le nombre modéré d'arriérés et/ ou de condamnations pose enfin la question du bon fonctionnement des structures du prélèvement. Les mentions sont-elles limitées, parce que les procédures l'étaient également? Les assujettis acceptaient-ils dans ses principes le prélèvement? En l'état actuel, les réponses ne peuvent trouver de conclusion ferme en dehors des études de cas.

\section{Les formes du prélèvement seigneurial}

Le prélèvement, en pays de Retz comme ailleurs, est caractérisé par une grande diversité de formes, comme le montre d'ailleurs la richesse du vocabulaire. Mais, derrière cette apparente multiplicité, sur quelles bases repose-t-il réellement? Porte-t-il davantage sur la production - est-il, à cette occasion, perçu plutôt en argent ou en nature? - que sur le travail?

42. Par exemple : " la some de dix sols de rente assis sur nos moulins neufx de Pornich et leur estre paiez le jour de la Nostre Dame meaougst, par chacun an, par la main de nostre dit chastelain ou receveur. " Arch. dép. de Loire-Atlantique, E 80.

43. $C f$. note 41 .

44. Les actes transmis ne permettent pas de saisir les sanctions encourues en cas de non respect.

45. Par exemple : "Et cognurent celles parties esdiz noms que sur le débat qui estoit entreulx esdiz noms sur le fait et débat de tres soulz de rente que avoit dit et disoit Boutin oudit nom que ledit deffunt devoit et avoit fait possession auxdiz abbé et couvant et à leurs prédicessours. Celx consors avoint voulu et promis et de fait promistrent et se obligèrent paier et rendre aux diz abbé et couvant et à leurs successours le numbre de troys soulz de cens ou rente ", extrait d'un accord conclu le 8 novembre 1405 entre les moines de Buzay et Guillaume Pillet (Arch. dép. de Loire-Atlantique, H 34). 


\section{Les redevances fixes}

Les redevances fixes portent toutes sur les terres ou les maisons concédées. Généralement placées sous le régime de la censive ${ }^{46}$, les tenures sont d'abord soumises à des redevances en argent. Modestes pour les paysans ${ }^{47}$, leur part varie dans les recettes totales en fonction du rang de leur détenteur. Les châtelains, par leur position et leur autorité, occupent sans conteste le sommet de la hiérarchie en constituant de véritables relais entre les sires et les seigneurs inférieurs. L'exercice de cette autorité donne lieu à la perception d'autres droits, souvent plus lucratifs. Les redevances fixes pèsent par conséquent peu dans les revenus totaux. Les comptes des Huguetières ou de Machecoul révèlent leur faible rapport comparé aux revenus des fermes ou des banalités ${ }^{48}$. Les redevances fixes occupent une part plus importante pour les structures seigneuriales inférieures ${ }^{49}$, bien qu'il soit impossible de connaître les revenus totaux des seigneuries ${ }^{50}$.

Les redevances fixes sont aussi une marque de l'autorité seigneuriale, à l'instar du cens. Redevance première de la tenure, à laquelle il a donné son nom, le cens est, en théorie, fixé une fois pour toutes et ne peut être révisé tant que demeurent le bénéficiaire du bail initial et ses héritiers, même lointains. Il distingue la propriété utile, exploitée par le paysan qui s'acquitte pour cela d'une rente, de la propriété éminente, symbolique, qui revient au seigneur ${ }^{51}$. En distinguant l'usage de la possession du sol, le cens met donc en œuvre une double propriété pour un même fonds. Même faible, le cens est toujours exigé, car il symbolise le maintien de l'autorité seigneuriale sur la terre concédée.

Les seigneurs imposent d'autres redevances fixes en nature pour compléter celles en argent ${ }^{52}$. Les censives, déjà soumises aux " rentes certaines

46. Une ambiguïté peut demeurer, car les rédacteurs ne précisent pas toujours la nature de la concession.

47. «Item, au terme de Pasques, à cause de ladicte censive, deiz deniers de rente. Item, à cause dicelle censive, par chacun an, au terme de meoust, seix soulz de rente. ", extrait de l'aveu rendu le 14 août 1426 par Perrot Maillart (Arch. dép. de Loire-Atlantique, 1 E 217).

48. Les redevances fixes procurent entre un tiers et un cinquième des recettes totales suivant les années : RАВОт, Brice, Les structures seigneuriales..., op. cit., graphique ${ }^{\circ} 1$, p. 105.

49. Ibidem, graphique $\mathrm{n}^{\circ}$ 2, p. 106 .

50. Les comptes n'indiquent pas les revenus perçus directement par les seigneurs : « Et ne compte pas ce receveur de la ferme de la levée de l'erbe du pré du Grant Faucheis, du Petit Faucheis, du pré Coquinet, ne du pré à la Chevallière dudit an mil IIIIc XxxvI, pour ce que monseigneur des puis les a vendus à Perrin Quili, celui an, et en a receu le poiement. " Arch. dép. de Loire-Atlantique, 1 E 221 (4), $\mathrm{f}^{\circ} 4 \mathrm{r}^{\circ}$.

51. Ce que les sources désignent sous l'expression " obéissance comme homme estaigier et doit à son seigneur et selont que le fié et les tenues le requièrent ", extrait de l'aveu rendu par René de Quersy devant la cour du Pellerin le 14 mars 1483 (n. st.) (Arch. dép. de Loire-Atlantique, B 1829).

52. Par exemple : « de Guillaume Seignorin, de la paroisse de Saint-Michel-de-Chevecher [aujourd'hui : de Chef-Chef], dix huit deniers et ung boisseau de seigle de rente, à paiez par la main dudit Seignorin chascun an au jour de la my aoust ", extrait de l'aveu rendu 
en deniers ", font l'objet de prélèvements limités. Les rentes exigées portent le plus souvent sur le froment ${ }^{53}$, sans que le prélèvement soit représentatif de l'orientation des cultures. Le froment n'est pas la céréale la plus exploitée : les seigneurs exigent les redevances en froment pour alimenter leur grenier ou pour pouvoir ensuite échanger les quantités reçues sur les marchés extérieurs. Le seigle ${ }^{54}$ et l'avoine ${ }^{55}$, sur lesquels portent d'autres taxes, sont en réalité davantage cultivés, même si les superficies totales qui leur sont consacrées demeurent inconnues. Destinés à l'alimentation des chevaux ou aux échanges extérieurs, le seigle et l'avoine font l'objet de prélèvements moindres que le froment ${ }^{56}$. Quelques rentes en baillorge ${ }^{57}$ peuvent, à l'occasion, être aussi levées ${ }^{58}$; elles sont en fait beaucoup plus rares car le baillorge sert surtout à répondre aux besoins des familles paysannes qui la cultivent sur les terres moins fertiles. Les seigneurs n'imposent donc que de faibles droits. Au total, les redevances fixes pèsent beaucoup moins sur les tenanciers que les terrages par exemple. Les redevances fixes en nature ne sont pas non plus liées à un type de structure seigneuriale. Elles varient, en revanche, selon les types d'exploitation agricoles. Les censives, par exemple, très répandues en pays de Retz, même si la situation évolue fortement dans les dernières décennies du $\mathrm{XV}^{\mathrm{e}}$ siècle, conduisent les seigneurs à exiger plutôt des rentes en argent que des rentes en nature.

La plupart des aveux présentent aux côtés des redevances précédentes quelques volailles; les quantités cumulées compensent en partie la faiblesse du prélèvement au départ. Les receveurs des Huguetières lèvent ainsi en moyenne chaque année 16 chapons ${ }^{59}$, tandis que ceux de La Blanchardais perçoivent 57 chapons et 6 gélines ${ }^{60}$. Ces volailles servent avant tout à garnir la table seigneuriale; quelques-unes sont reversées à d'autres receveurs au titre des reconnaissances de fief. Les prélèvements demeurent

par Perrot de Vauze le 27 juin 1401 (Cartulaire des sires de Rays..., op. cit., pièce XLVIII, p. 120-121).

53. Pour les seigneurs de La Blanchardais, par exemple, les recettes en froment fournissent la moitié des prélèvements en nature totaux (RABOT, Brice, Les structures seigneuriales..., op. cit., p. 449-450).

54. Par exemple : Arch. dép. de Loire-Atlantique, B 1859 (Saint-Brévin et Saint-Père-enRetz), B 1866 (Sainte-Opportune-en-Retz), B 1870 (Machecoul), E 150 (Saint-Colomban), E 362 (Port-Saint-Père), 1 E 217 (Vue) (RABOT, Brice, Les structures seigneuriales..., op. cit., p. 210).

55. Par exemple : Arch. dép. de Loire-Atlantique, B 1829 (Le Pellerin), B 1836 (SaintLumine-de-Coutais), B 1870 (Paulx, Saint-Philbert-de-Grand-Lieu, Saint-Mars-de-Coutais, Saint-Même-le-Tenu), Arch. dép. de Loire-Atlantique, 1 E 217 (Vue); RABOT, Brice, Les structures seigneuriales..., op. cit., p. 210.

56. Les bilans des comptes de La Blanchardais ou des Huguetières sont particulièrement révélateurs.

57. Autre nom donné à l'orge de printemps (ou baillarge).

58. Par exemple, pour Les Huguetières (Arch. dép. de Loire-Atlantique, E 501 (1, 3, 4 et 5), E 502 [2 et 3]).

59. SADDIER, Évelyne, Une seigneurie rurale..., op. cit., p. 46.

60. Arch. dép. de Loire-Atlantique, 1 E 222 (1), $\mathrm{f}^{\circ} 15 \mathrm{v}^{\circ}-16 \mathrm{r}^{\circ}$ 
en tout cas très modestes ${ }^{61}$ et ont une portée plus symbolique qu'économique. Les profits tirés des rentes en volailles apparaissent somme toute faibles, surtout lorsqu'on les compare à ceux des fermes des glandées et des panages ${ }^{62}$.

\section{Les redevances partiaires}

Les redevances partiaires sont " d'une pratique courante ${ }^{63}$ " dans l'Ouest de la France au Moyen Âge, à l'exception de la Bretagne où elles demeurent très limitées ${ }^{64}$. La concentration des terrages et des complants, sans oublier les dîmes, rarement mentionnées, constitue l'une des principales originalités du pays de Retz par rapport au reste du duché ${ }^{65}$. Comment expliquer une telle situation? Les redevances partiaires sont-elles liées à l'autorité seigneuriale plus forte de la baronnie de Retz? Ne sont-elles pas plutôt le reflet des influences extérieures, qui orientent aussi les structures agraires, dans les zones de marches notamment? Les réponses varient selon les types de redevances.

Les dîmes et les terrages figurent parmi les plus répandues. Parfois appelées "menues dîmes ${ }^{66}$ ", les dîmes reposent sur les produits de la terre et de l'élevage et représentent, en général, un dixième des revenus paysans ${ }^{67}$. Très souvent confondues avec les autres redevances foncières, les dîmes sont directement perçues par les seigneurs laïcs ou leurs agents ${ }^{68}$. Les ambiguïtés demeurent d'autant plus grandes ${ }^{69}$ que les rédacteurs ne

61. Entre 1456 et 1461, par exemple, sur les 403 chapons et 36 poules perçus par le receveur de Vue, 292 chapons et 35 poules sont reversés à ce titre (Arch. dép. de LoireAtlantique, 1 E $222[1], \mathrm{f}^{\circ} 28 \mathrm{v}^{\circ}$ ).

62. Les comptes des Huguetières indiquent des revenus annuels pouvant s'élever jusqu'à 80 livres pour le panage et la glandée de cheptels importants : 1658 porcs sont ainsi mis en glandée dans la forêt de Machecoul et 329 dans celle de La Chevrolière en 1426 (Arch. dép. de Loire-Atlantique, E 502 [1], f $7 \mathrm{r}^{\circ}-8 \mathrm{v}^{\circ}$ ); SADDIER, Évelyne, Une seigneurie rurale..., op. cit., p. 67 .

63. LE MenÉ, Michel, " Les redevances à part de fruits dans l'Ouest de la France au Moyen Âge ", dans Les revenus de la terre, complant, champart, métayage en Europe occidentale (IX ${ }^{e}$-XVIII ${ }^{e}$ siècles) (Flaran, 7), Auch, 1985, p. 9.

64. Ibidem, p. 11-13. RАВОт, Brice, Les structures seigneuriales..., op. cit., p. 209-218.

65. RАВот, Brice, Les structures seigneuriales..., op. cit., p. 204-221.

66. Arch. dép. de Loire-Atlantique, E 362 (Port-Saint-Père), E 489 (La Bénâte), E 522 (Machecoul).

67. "Item les deux pars du dixiesme des laynes, aygneaux, blez, potages et autres choses croissans chacun an esdictes choses. Item le terrage et les deux pars du dixiesme \{en sept\} boexellées de terre ou environ que Olivier Thébauld tient entre lesdictes bones derroinement nomées. ", extrait de l'aveu rendu le 2 janvier 1453 (n. st.) par Jehan Le Rasle (Arch. dép. de Loire-Atlantique, B 1866).

68. "Item, vignt et deux hommées de vigne et le quart de la levée de quatre hommées de vigne et les deux pars de la disme desdictes quatre hommées de vigne, la levée desquelles choses valit l'an du déceix dudit deffunct le tiers d'une pippe de vin " : extrait du minu rendu le 10 octobre 1428 par Guillemete Guienne (Arch. dép. de Loire-Atlantique, B 1866).

69. "Item, le devoir de terrage que nous avons sur quinze hommées de vigne et ouict boexeaux de terre laborable, siises en la paroesse de La Pleine, et les deulx pars des 
prennent pas toujours soin de distinguer les différentes redevances ${ }^{70}$. Les taux, parfois proches ${ }^{71}$, les modalités de perception, jamais clairement indiquées, entretiennent le doute. Les terrages font l'objet d'indications plus précises. Portant sur les produits de la terre ${ }^{72}$, ils sont loin d'être uniformes avec de très fortes variations selon les cultures et les coutumes (figure 1). Les taux relevés en pays de Retz figurent parmi les plus lourds connus en Bretagne méridionale ${ }^{73}$.

Les taux les plus faibles, du dixième au onzième des récoltes ${ }^{74}$, reposent sur les tenures céréalières déjà soumises aux redevances fixes. Ajoutés par les seigneurs, les terrages offrent ainsi quelques compléments. Les rendements peu élevés, bien qu'impossibles à connaître dans le détail, modèrent aussi les exigences. Les terrages sont en revanche beaucoup plus lourds pour les tenures lorsque d'autres redevances ne sont pas exigées ${ }^{75}$. Cette dualité explique la multiplicité des taux de terrages, qui peut d'ailleurs s'exercer à l'échelle d'une même paroisse ${ }^{76}$. Le pays de Retz n'est pas non plus le seul exemple dans l'Ouest à offrir une telle dualité. Deux systèmes de terrages coexistent aussi en Anjou dès le XIII ${ }^{\mathrm{e}}$ siècle ${ }^{77}$, avec des taux plus lourds pour les tenures qui n'acquittent pas d'autres redevances. La proximité géographique, les similitudes observées entre les systèmes développés en Anjou ou en Poitou et en pays de Retz montrent le poids des influences extérieures dans l'orientation des structures du prélèvement.

dismes sur lesdictes chouses, sauff que le prieur de Roaut y prant le onziesme sur lesdictes dismes ", extrait de l'aveu rendu aux moines de Blanche-Couronne en 1485 (Arch. dép. de Loire-Atlantique, H 17).

70. C'est ce qu'indique l'aveu rendu par René de Rays le 27 août 1462 : «O touz les drois, prérogatives, previlèges et noblesses que en déppendent et pevent déppendre [...] prés, rentes en deniers, blez, vins, poulailles, dismes, terraiges, biams, courvées, coustumes, cohuaiges, foires, marchés, portz, havrez, bris et nauffraiges de mer, espavez, galoiz, droit de bailler, brieufs de mer" (Arch. dép. de Loire-Atlantique, B 1854).

71. RАвот, Brice, Les structures seigneuriales..., op. cit., p. 213-217.

72. "Item la moytié de l'onzain des blez croessanz par labour en une tenue de terres, nomée Chasteillon, en la parroesse de Sainct-Mars-de-Costays, par indivis o Jehan Meschinot, seigneur de La Haie du Coustais, et qui exeguent les terrages et prant en la moitié et appartenances audit Gouy la septiesme partie et le persus fait celui Meschinot randre audit Gouy ledit septiesme rabbatu. Et à cause de ce doibt ledit Meschinot audit Gouy par chacun an le mercredi avant Noël cinquante chieffs d'aill et une petite mesure de moustarde randu en la ville de Machecoul. Quelx terrages et choses dessus dictes pevent valler communs ans trois boexeaux de blé vallens seigle ", extrait de l'aveu rendu le 13 décembre 1455 par Sevestre Gouy (Arch. dép. de Loire-Atlantique, B 1870).

73. Certains s'élèvent jusqu'au tiers des productions. Par exemple, dans la région de Saint-Philbert-de-Grand-Lieu (Arch. dép. de Loire-Atlantique, B 1870).

74. Arch. dép. de Loire-Atlantique, B 1816, B 1870.

75. «Item, que ledit Guoy a et prant par terrage en ladicte parroaisse de Saint-Mars, en ung fié et tènement nommé la Druillarderie et Lavugnes, le terrage des blez y croissans, quel terrage se liève au seixte ouquel seixte ledit Guoy prant une moitié et la moitié en l'autre moitié ", extrait de l'aveu rendu le 31 janvier 1444 (n. st.) par André Guoy, seigneur de la Noé Pourceau et du Branday (Saint-Philbert-de-Grand-Lieu) (Arch. dép. de LoireAtlantique, B 1870).

76. Par exemple, à Saint-Philbert-de-Grand-Lieu (Arch. dép. de Loire-Atlantique, B 1870).

77. LE MENÉ ", Michel, "Les redevances... ", loc. cit., p. 14-15. 


\section{Figure 1 - Répartition spatiale des mentions de terrages et de complants en baronnie de Ret $^{78}$}

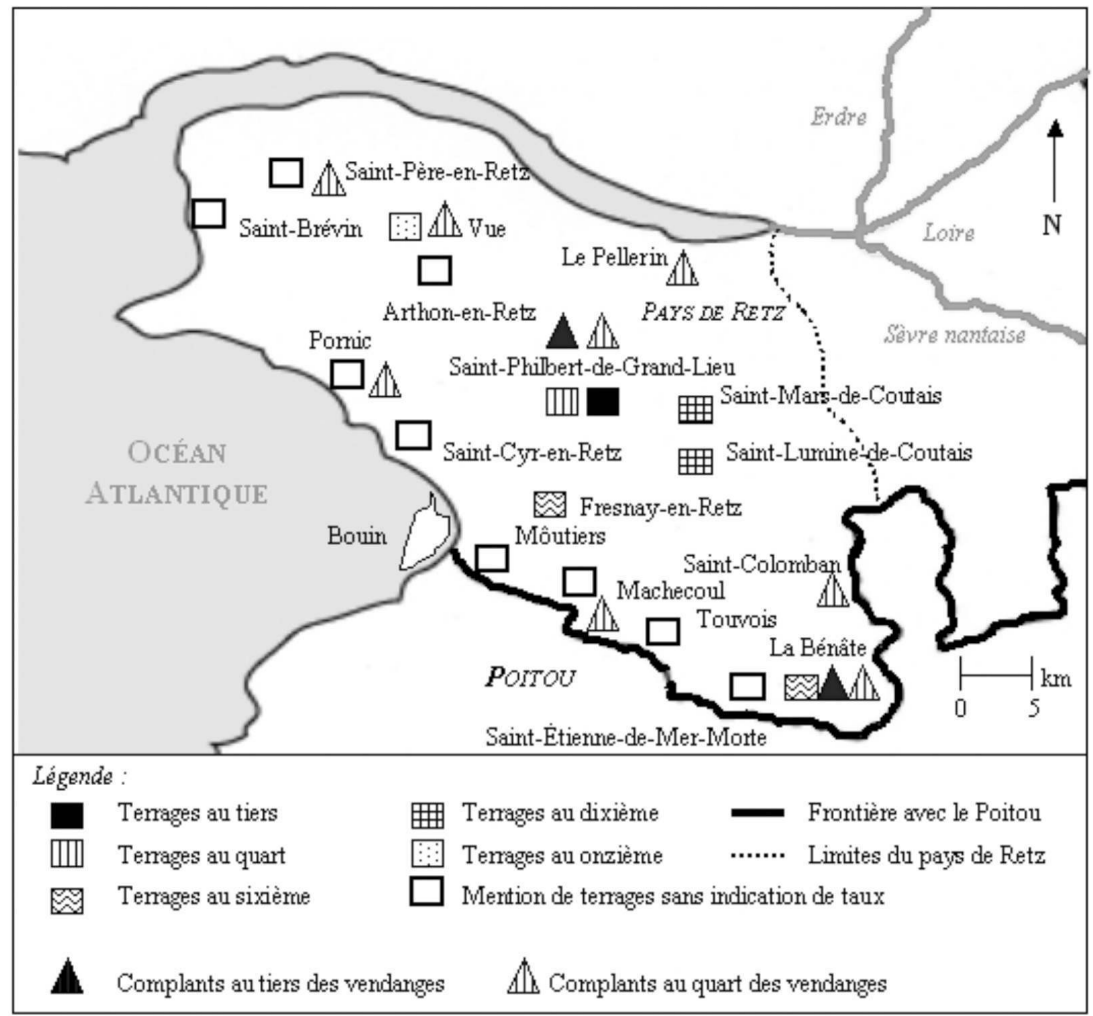

Les redevances partiaires ne s'exercent pas seulement sur les céréales, mais aussi sur la vigne avec le système du complant, particulièrement répandu en pays de Retz. Conclu entre un bailleur qui abandonne sa terre et un cultivateur qui se charge de la planter en vigne, selon les modalités prescrites dans un bail, le complant est une sorte de pariage qui vise à développer la viticulture ${ }^{79}$. Les revenus, très élevés, conduisent les seigneurs

78. D’après (terrages) : Arch. dép. de Loire-Atlantique, B 842, B 843, B 1836 (SaintLumine-de-Coutais), B 1816, E 490 (La Bénâte), B 1854 (baronnie de Retz); B 1870 (SaintPhilbert-de-Grand-Lieu), B 1875 (Port-Saint-Père), E 522 (Machecoul), E 607 (Saint-Pèreen-Retz), 1 E 217 (La Blanchardais); d'après (complants) : Arch. dép. de Loire-Atlantique, B 1816, E 490 (La Bénâte), B 1859 (Saint-Père-en-Retz), B 1870 (Saint-Philbert-de-GrandLieu), E 352, E 368 (Port-Saint-Père), E 522 (Machecoul), E 609 (Sainte-Opportune), 1 E 217 (Vue), 1 E 746 (Saint-Colomban), H 10 (Pornic), H 39 (Le Pellerin).

79. Gallet, Jean, Seigneurs et paysans bretons du Moyen Âge à la Révolution, Rennes, Éditions Ouest-France Université, 1992, p. 224; PlanIOL, Maurice, Histoire des institutions de la Bretagne (t. 4 : La Bretagne ducale), Mayenne, 1982, p. 261-268. 
à exiger des parts de vendange pouvant s'élever au tiers ${ }^{80}$ ou au quart des productions ${ }^{81}$, auxquelles s'ajoutent des redevances en $\operatorname{argent}^{82}$. La proximité des voies de communication et du marché nantais ${ }^{83}$ favorise également le développement des complants, en limitant les coûts de transport et en offrant des débouchés en Bretagne ou à l'extérieur. Les complants entretiennent aussi des rapports étroits avec les terrages ${ }^{84}$ au point qu'ils sont parfois confondus. Les modalités de perception et les taux identiques entraînent là encore une ambiguïté que les rédacteurs entretiennent.

Les complants portent aussi la trace des influences extérieures. Les liens entretenus avec le Poitou expliquent leur forte implantation en pays de Retz. Jusqu'au milieu du $\mathrm{x}^{\mathrm{e}}$ siècle, au moment où ils commencent justement à se répandre, le pays de Retz se trouve placé sous la domination poitevine. Les seigneurs y développent alors les complants comme dans le reste du Poitou. Dans la seconde moitié du $\mathrm{X}^{\mathrm{e}}$ siècle, le rattachement définitif du pays de Retz à la Bretagne n'interrompt pas le processus ${ }^{85}$. Le maintien de relations étroites, dans les zones de marches communes en particulier ${ }^{86}$, soutient les initiatives et les entreprises déjà engagées. Les complants continuent d'ailleurs de progresser, avec les nouvelles baillées ${ }^{87}$,

80. Par exemple : Arch. dép. de Loire-Atlantique, B 1819.

81. Les complants s'élèvent généralement au quart des vendanges en pays de Retz : LE MENÉ, Michel, "Les redevances... ", art. cit., p. 20-21.

82. Le " denier de pevage " ou " vignage " est fréquemment exigé en sus de la part de vendange : " Item, le quart de la vendenge et la disme qui croist par an es fiez des vignes des Pantonardères, siis en la parroaisse de Saint-Philibert-de-Grant-Lieu, qui contiennent ouyt vignts hommées de vigne ou environ. Item, ung denier de chacune hommée de vigne et deux deniers de pas de chacun labouroux au terme de vendenges ", extrait de l'aveu rendu le 31 janvier 1444 (n. st.) par André Guoy (Arch. dép. de Loire-Atlantique, B 1870).

83. GUIBERT, Alain, Étude sur la vigne dans le comté nantais au Moyen Âge, mémoire de DES (dactyl.), Université de Nantes, 1970, p. 29. Pour une remise en perspective du commerce du vin dans les échanges et les revenus que les seigneurs pouvaient en retirer : Renouard, Yves, "Le grand commerce du vin au Moyen Âge ", Revue historique de Bordeaux, 1952, p. 5-18.

84. Cf. note 15. La présence de complants à proximité, notamment à Pornic, ne permet pas d'exclure leur présence à La Plaine-sur-mer, même si l'aveu en question n'en fait pas état.

85. Les complants sont alors placés sous l'autorité de la coutume : FougERoux, Ernest $\mathrm{du}$, "Question de droit historique : la vigne à complant en Poitou et en Bretagne ", Revue de Bretagne et de Vendée, t. 15, 1864, p. 283.

86. Les marches communes, autrement dit les zones dans lesquelles les seigneurs poitevins et bretons exercent, en théorie, conjointement leur autorité de part et d'autre de la frontière, se répartissent en deux zones autour de La Bénâte à l'est et de Bouin à Paulx à l'ouest (CinTRÉ, René, Pichot, Daniel, « Les "marches séparantes de Bretagne, Poitou et Anjou" et la frontière orientale ", dans LAGRÉE, Michel, TANGUY, Bernard (dir.), Atlas d'histoire de la Bretagne, Morlaix, Skol Vreizh, 2003, p. 67).

87. Par exemple, à La Limouzinière : "C'est assavoir une pièce de terre contenant quatre charruez de terre ou environ pour planter et commectre en vigne la dicte pièce, sise entre les prez de l'Uguetière et le Pouceau et le chemin par ou l'on vait du Puy Millon à Sainct-Columbam d'une part et d'autre ", extrait du bail à complant conclu le 20 mars 1394 (n. st.) entre Jehan Rortay le jeune et Johan de La Tousche (Arch. dép. de LoireAtlantique, 1 E 746). 
pour aboutir à la situation observée à la fin du Moyen Âge avec une très forte concentration en pays de Retz.

\section{Les corvées}

Le prélèvement ne porte pas uniquement sur les productions agricoles. Il porte aussi sur le travail paysan avec les corvées ou les " bians ${ }^{88}$ ". Encore faut-il distinguer les services dus pour le seigneur foncier (pour la réserve) des services dus pour le seigneur banal. Leur poids et leurs modalités ne sont en effet pas tout à fait identiques. Comme ailleurs, les sources renseignent davantage sur la seigneurie banale que sur le fonctionnement interne de la seigneurie foncière. Les corvées les moins lourdes reposent, sans conteste, sur les maisons ou les terres concédées; une à deux journées de labeur leur sont en moyenne consacrées chaque année, essentiellement pour des travaux agricoles ${ }^{89}$. L'exploitation des ressources de la réserve donne lieu à des corvées beaucoup plus lourdes. À La Gayne (Machecoul), les paysans doivent fournir, tous les quinze jours, deux bœufs et une charrette pour transporter le bois coupé depuis les forêts de la réserve ${ }^{90}$.

Ces différences pour les assujettis posent la question de la valeur économique des corvées. Ont-elles d'ailleurs toujours principalement une valeur économique? N'ont-elles pas plutôt une valeur symbolique? Les comptes apportent à ce sujet quelques éléments de réponse. Les ressources tirées des corvées sur les tenures sont limitées ${ }^{91}$, tandis que celles de la réserve sont beaucoup plus lucratives ${ }^{92}$. Les premières sont davantage liées à l'affirmation de l'autorité symbolique du seigneur, en contraignant les tenanciers

88. Par exemple : Arch. dép. de Loire-Atlantique, E 150 (Saint-Colomban), E 522 (Machecoul). Les bians désignent la plupart du temps des corvées rachetables par les assujettis.

89. Par exemple : "Item, deulx soulx de rente que me doit Macé Thébaudeau sur ung courtil siis près les terceries et ce que je ay et prans en la Prévocerie et en l'Angevinière, tant en blez, deniers de rentes que aultres devoirs et quarente sols de rente que me devent les Charbonneaulx et une forche de (bien) biam que me devent lesdiz hoirs asenez les prez appellez les prez de Lacharrau ", extrait de l'aveu rendu pour rachat le 12 décembre 1451 (Arch. dép. de Loire-Atlantique, E 150).

90. "Item, d'un aultre devoir appellé bian deu chacun an en la chastelainie dudit lieu Machecoul par les tenuers du fye de La Gayne de quinze jours en quize [sic] jours, une journée o deux beufs et une charrecte pour amener au chastelain de Machecoul les boys de ladicte seigneurie, affermé celui debvoir pour l'an de ce compte à Jehan Delorme, comme plus donneur et derroin enchérisseur à la somme de 4 livres 5 soulz, comme aparoit par le précédent de ladicte baillée, datée le 25 jour de janvier l'an mil IIIIc IIIIxx XVII. Passe O. Legrant et Jac Berthelot, s'en charge ledit receveur pour ledit an 4 livres 5 soulz. " Arch. dép. de Loire-Atlantique, E 522 (2), $\mathrm{f}^{\circ} 3 \mathrm{v}^{\circ}$.

91. Le cumul des corvées compense en partie la faiblesse de départ. Leur produit ne peut pas être quantifié, faute d'éléments chiffrés.

92. Les enchères doivent permettre d'accroître leur valeur. Les registres permettent d'ailleurs d'établir quelques comparaisons avec d'autres enchères. Ainsi, à Machecoul, en 1461, la ferme du péage est emportée pour 60 livres 12 sous 6 deniers, tandis que celle du charroi des bois de la réserve (corvée) est négociée, en 1463, 68 livres 9 deniers (Arch. dép. de Loire-Atlantique, E 522 [1], $\mathrm{f}^{\circ} 5 \mathrm{r}^{\circ}, 9 \mathrm{v}^{\circ}$ ). 
à effectuer certaines tâches ou à s'acquitter de sommes, même modestes, tandis que les secondes sont beaucoup plus importantes, en termes de revenus, car les profits attendus de la vente des bois sont aussi plus élevés.

Les corvées sont d'autant plus pénalisantes qu'elles s'ajoutent aux travaux des exploitations, à des moments où les travaux agricoles nécessitent la mobilisation de toutes les forces. Elles induisent également des dépenses supplémentaires : la fourniture des montures et des attelages, pour les charrois par exemple, reste à la charge des assujettis ${ }^{93}$. Les modalités pour les autres corvées demeurent plus incertaines. Sans doute les paysans devaient-ils se rendre directement avec leurs propres outils.

Les corvées ne s'avèrent pas, au total, trop contraignantes pour les paysans du pays de Retz. Elles portent davantage la marque, comme les autres redevances, de l'autorité seigneuriale et rappellent l'obéissance due au seigneur foncier ${ }^{94}$. Leur modestie peut enfin être interprétée comme la difficulté, pour la seigneurie, d'encadrer des hommes largement dispersés.

\section{Les banalités}

L'obligation évidente ${ }^{95}$ et la nécessité pour les paysans de fréquenter les moulins, fours ou pressoirs seigneuriaux font de ces édifices des piliers de l'encadrement seigneurial, en particulier dans le contexte de dispersion des hommes et des biens ${ }^{96}$. Le pays de Retz concentre une forte densité d'édifices banaux, révélatrice de l'emprise seigneuriale ${ }^{97}$. Les seigneurs de La Blanchardais disposent du patrimoine le plus réduit avec un four, en plein bourg de Vue, complété par deux moulins ${ }^{98}$. Les moines de Buzay détiennent de nombreux moulins et des pressoirs au Pellerin, à Pont-Saint-

93. « Du proufit du biam de La Gayne qui est deu de quinze jours en quinze jours par les tenuers du fye de La Gayne o deux beufs et une charrete n'en compte riens ce receveur avoir receu du temps escheu depuix la fin de son derrain compte jucques au premier jour de febvrier l'an mil IIIIC LXII par ce qu'il a \{esté\} esplecté au chastelain dudit Machecoul et affermés de la maison durant ledit temps. " Arch. dép. de Loire-Atlantique, E 522 (1), $\mathrm{f}^{\circ} 9 \mathrm{v}^{\circ}$.

94. Les corvées liées à l'arbitraire seigneurial sont très rares. Aux confins du pays de Retz, Hubelin Chasteignier, seigneur à Bouguenais, fixe librement la date et la personne chargée d'effectuer la corvée : "Item, une baillée de seune une foiz l'an en l'isle de Botie, estante en ladicte parroesse de Saint-Père-de-Bouguenays, à tieul jour et heure et en tieulle saezon qu'il plaest audit chevalier choaesir et eslire ", extrait d'un aveu rendu pour rachat le 25 août 1451 (Arch. dép. de Loire-Atlantique, B 1819).

95. "Et en oultre fut et est ledit Chace confessant qu'il doit faire mouldre par destroit ses blez au molin à cause dudit Guillaume Blanchart faire les porter ne faire porter à autres molins delà où ledit molin sera en point de mouldre et qu'il sera moullant. ", extrait de l'aveu rendu le 21 mai 1416 par Perrot Chace (Arch. dép. de Loire-Atlantique, 1 E 217).

96. Piснот, Daniel, "Le moulin et l'encellulement dans l'ouest français (XI ${ }^{\mathrm{e}}$-XIII ${ }^{\mathrm{e}}$ siècles) ", dans Flaran 21, Moulins et meuniers dans les campagnes européennes (IX ${ }^{e}$-XVIII $e^{e}$ siècles), Toulouse : Presses Universitaires du Mirail, 2002, pp. 111-129; RABOT, Brice, Les structures seigneuriales..., op. cit, p. 228.

97. RАвот, Brice, Les structures seigneuriales..., op. cit., carte $\mathrm{n}^{\circ} 6$, p. 228.

98. Arch. dép. de Loire-Atlantique, 1 E $222(1), \mathrm{f}^{\circ} 4 \mathrm{r}^{\circ}-\mathrm{v}^{\circ}$. 
Martin, à Bouin, à Saint-Philbert-de-Grand-Lieu ou encore à La Bénâte ${ }^{99}$. Les seigneurs des Huguetières entretiennent des moulins à La Chevrolière (Haux-Champs), à Saint-Philbert-de-Grand-Lieu (La Chaussée, La Motte) ainsi qu'à Saint-Colomban (Pont-James) ${ }^{100}$. Les autres patrimoines saisissables sont beaucoup plus clairsemés ${ }^{101}$, du fait de richesses et d'autorité plus réduites.

Placés sous l'autorité d'un seigneur, qui délègue le plus souvent leur gestion à un receveur, ces édifices reflètent, par leur empreinte dans le paysage, la marque de la seigneurie banale. Ce poids ne doit cependant pas être surestimé. L'historiographie récente conduit à reconsidérer le rôle et la participation des communautés rurales dans la construction ou l'entretien des moulins ${ }^{102}$. Loin d'être passives, elles jouent en effet un rôle fondamental, en particulier aux $\mathrm{x}^{\mathrm{e}}$-XII ${ }^{\mathrm{e}}$ siècles, en rendant possible, par leur travail, l'établissement et le fonctionnement des moulins. Aux XIV et XV ${ }^{\mathrm{e}}$ siècles, les paysans continuent de jouer un rôle de premier plan en se portant acquéreurs des fermes pour exploiter les fours ou les moulins ${ }^{103}$. Les receveurs préfèrent en effet affermer les prélèvements, contre des paiements en nature ${ }^{104}$ ou en argent ${ }^{105}$, plutôt que de les lever directement lors de chaque utilisation. Les fermiers deviennent ainsi des rouages indispensables du prélèvement, permettant au receveur de se consacrer à d'autres tâches.

Les édifices banaux sont aussi très importants pour les tenanciers. En leur permettant de transformer leurs denrées de base en produits semifinis, plus facilement négociables et transportables, les moulins, les fours et les pressoirs jouent un grand rôle dans l'économie rurale en participant à la prospérité de la communauté paysanne. Ils peuvent donc être considérés non plus comme des outils de contrainte, imposés aux paysans par les seigneurs qui n'en tirent d'ailleurs pas forcément d'importants revenus du fait des coûts d'entretien ${ }^{106}$, mais bien comme des outils mis à la dispo-

99. Arch. dép. de Loire-Atlantique, H 39.

100. SADDIER, Évelyne, Une seigneurie rurale..., op. cit., p. 10.

101. Par exemple, pour la châtellenie de Pornic : " ladicte parroesse de Chauvrié, entre [...] La Rigaudière, La Hardière, le molin neuff et le Par Boceau d'une et aultres parties. ", extrait d'un aveu rendu le 11 mars 1458 (n. st.) par la veuve de Jacquet de Béac (Arch. dép. de Loire-Atlantique, B 1829).

102. ARnoux, Mathieu. Le temps des laboureurs. Travail, ordre social et croissance en Europe (XI ${ }^{e}-X I v^{e}$ siècle), Paris, Albin Michel, 2012, p. 336.

103. Les noms retranscrits dans les registres des Huguetières ou de La Blanchardais montrent qu'il s'agit des tenanciers exploitant les terres des paroisses alentours (Arch. dép. de Loire-Atlantique, 1 E 221 à 223 [La Blanchardais], E 500 à 503 [Huguetières]).

104. Par exemple, pour Les Huguetières, 7 setiers et 14 boisseaux de seigle en 1409 (Arch. dép. de Loire-Atlantique, E 500 [6], f ${ }^{\circ} 9 \mathrm{v}^{\circ}$ ).

105. Par exemple, pour La Blanchardais, 3 livres 5 sous en 1431 (Arch. dép. de LoireAtlantique, 1 E 221 [1], $\mathrm{f}^{\circ} 4 \mathrm{r}^{\circ}$ ).

106. Aucun élément chiffré n'est fourni à ce sujet dans les sources disponibles pour le pays de Retz. Nous savons par ailleurs que certains travaux d'entretien s'avéraient particulièrement lourds, comme le remplacement des meules. Une meule revenait, en moyenne, à 30 ou 40 livres, auxquelles s'ajoutait le coût du transport et de la pose. De telles tâches nécessitaient des personnes qualifiées ou du moins suffisamment précises 
sition des tenanciers ${ }^{107}$. Cette réorientation des perspectives correspond au portrait dressé par les sources disponibles, les édifices banaux n'ayant pas fait l'objet de contestations en pays de Retz.

\section{Les modalités du prélèvement seigneurial}

L'étude du prélèvement conduit enfin à poser la question de l'organisation concrète, matérielle et humaine, de la perception. Sur quels appuis repose-t-elle et quelle place chacun occupe-t-il dans sa mise en œuvre?

\section{Les officiers}

Agents indispensables pour faire régner l'ordre et pour incarner l'autorité sur le terrain, les officiers sont, pour les seigneurs, indispensables dans la perception des rentes et des autres droits. Ils n'apparaissent pourtant qu'à de rares occasions dans les sources. Les comptes, avec la rubrique consacrée aux mises et paiements, s'avèrent une fois encore précieux pour établir une hiérarchie des officiers, selon les fonctions occupées et les gages perçus.

Le receveur est de loin le plus important des officiers. Représentant de l'autorité seigneuriale par excellence, interlocuteur privilégié et percepteur des rentes portables, le receveur est celui qui organise et permet la levée du prélèvement. Véritable régisseur, il accense les terres ou les biens en procédant aux nouvelles baillées. Son rôle se renforce tout au long du $\mathrm{XV}^{\mathrm{e}}$ siècle en lien avec les progrès accomplis en matière de gestion. Le receveur est aussi chargé des relations avec l'extérieur, soit pour défendre les intérêts de son maître, comme procureur ${ }^{108}$, soit pour s'acquitter, toujours au nom de son maître, des redevances pour les reconnaissances de fief ${ }^{109}$.

pour éviter que la meule ne soit mal fixée. Si l'horizontalité n'était en effet pas parfaite, l'usure était plus rapide, ce qui surenchérissait le coût d'entretien des moulins (LEGUAY, Jean-Pierre, MARTin, Hervé, Fastes et malheurs de la Bretagne ducale (1213-1532), Rennes, Éditions Ouest-France Université, 1997, p. 218).

107. ARnoux, Mathieu, Le temps des laboureurs..., op. cit., p. 221-255.

108. Les receveurs de La Blanchardais assistent régulièrement à des plaids judiciaires, ce qui donne lieu à des déplacements nombreux et coûteux : "Pour le sallaere de plusieurs journées que ce receveur, comme procureur de monseigneur en ses causes, a mis à aller et estre pour le deffandre es queulx lez et alloués de Nantes es plez de Rais, tant de Pornic que de Veuz, es plez de Bourcneuff et es plez des sires de Froczay, tant de La Hunaudaie, de Machecoul, que de Jehan Germaud à plusieurs termes et assignacions que monseigneur y a eu à besogner, tant les procureurs desdictes cours en deffance que o plusieurs aultres parties en faicte et en deffence pour les causes et négoce de mondit seigneur et auxi à plusieurs veages faire qui ne touchent pas le fait de la recepte ou ledit receveur a eu empeschement d'autant de temps et journées entendre à ses propres affères et esquelles pour lui lui a convenu mectre gens à les faire jucques au montement de IIc journées et plus, dont suplie à mondit seigneur et à messeigneurs les auditeurs de ces comptes sur ce lui en faire reson et mise à leur bon vouloir plésir et ordiennance. " Arch. dép. de Loire-Atlantique, 1 E 222 (3), $\mathrm{f}^{\circ} 28 \mathrm{v}^{\circ}$.

109. Par exemple : " À Jehan Foucaud, repceveur et chastelain de Rais à Veuz, a poié et baillé ledit receveur, pour rente deue chacun an au sire de Rays au terme de Noël, tes- 
Le receveur entretient donc des rapports privilégiés avec le seigneur, qui n'est d'ailleurs jamais très loin ${ }^{110}$.

D'autres officiers, aux fonctions plus spécialisées, épaulent le receveur. Le sénéchal est chargé d'organiser les cours de justice et de faire appliquer les grandes décisions. Assisté d'un alloué et, parfois, d'un procureur autre que le receveur, il représente lui aussi les intérêts du seigneur. Quelques sergents parcourent le territoire pour maintenir l'ordre, percevoir les amendes $^{111}$ et/ou prélever les rentes. Leur nombre exact demeure toutefois impossible à saisir. Les registres de La Blanchardais attestent aussi du recours à des censiers ${ }^{112}$, qui jouent un rôle plus étendu que la simple perception des rentes. Ils fournissent au receveur la liste mise à jour des tenanciers et des biens concédés, tâche indispensable pour pouvoir ensuite lever toutes les rentes foncières. Une dizaine de personnes suffit, en définitive, à faire vivre l'organisme seigneurial.

Les patrimoines plus étendus, à l'instar des Huguetières ${ }^{113}$, nécessitent des effectifs plus importants. Aux côtés du receveur figurent un sénéchal, un alloué, un procureur rétribué en moyenne 10 livres par an, qui occupent les mêmes tâches que précédemment. D'autres fonctions plus spécialisées apparaissent; un " ségreier ${ }^{114}$ " et un vendeur de bois sont chargés de sélectionner et vendre les bois, aidés de quatre personnes pour les bois de Machecoul et de deux autres pour ceux des Huguetières ${ }^{115}$, un sergent féal lève les rentes foncières ${ }^{116}$. Au total, une quinzaine de personnes animent, dans ce cas, l'organisme seigneurial.

Les officiers sont peu nombreux, car la plupart des rentes sont directement portées au manoir ou au grenier seigneurial par les tenanciers, à leurs

moign relacion dudit Foucaud, donnée le premier jour d'apvrill après Pasques l'an mil IIIIC LXXV 13 soulz 8 deniers [rayé et corrigé en : 4 deniers]. " Arch. dép. de Loire-Atlantique, 1 E $223(1), f^{\circ} 25 v^{\circ}$.

110. Par exemple, les seigneurs de La Blanchardais sont régulièrement présents lors des enchères : " Du prouffit et revenue de la ferme de la terre du pré à La Chevalière, du Grant Faucheis et du Petit Faucheis, affermé par ce receveur en la présence de monseigneur à Micheau Borreau, comme plus donnans, l'an 14619 livres. " Arch. dép. de LoireAtlantique, 1 E 222 (1), $\mathrm{f}^{\circ} 8 \mathrm{v}^{\circ}$.

111. " Des taux et amendes de la court et juridicion de monseigneur, ne s'en charge d'aucun prouffit du temps de ce compte, pour ce que le séneschal de la court de monseigneur, qui les a faiz, les a baillez à lever au sergent de ladicte court pour assigner de ses gages, ainsi que de coustume l'on a acoustumé de le faire. " Arch. dép. de Loire-Atlantique, 1 E $222(2), \mathrm{f}^{\circ} 12 \mathrm{r}^{\circ}$.

112. Arch. dép. de Loire-Atlantique, 1 E 222 (1), $\mathrm{f}^{\circ} 27 \mathrm{v}^{\circ}, 28 \mathrm{r}^{\circ} ; 1$ E 222 (3), $\mathrm{f}^{\circ} 32 \mathrm{r}^{\circ}$; 1 E 223 (1), $\mathrm{f}^{\circ} 26 \mathrm{r}^{\circ} ; 1 \mathrm{E} 223$ (2), $\mathrm{f}^{\circ} 31 \mathrm{r}^{\circ}$.

113. Bâtie autour du manoir de La Chevrolière, à $7,5 \mathrm{~km}$ au nord-est de Saint-Philbertde-Grand-Lieu, cette châtellenie dispose d'une base foncière élargie à 18 paroisses, entre Loire-Atlantique et Vendée actuelles (SADDIER, Évelyne, Une seigneurie rurale..., op. cit., p. 6).

114. Sergent forestier en charge de l'exploitation d'un secteur de forêt.

115. Arch. dép. de Loire-Atlantique, E 502 (3), $\mathrm{f}^{\circ} 20 \mathrm{v}^{\circ}$.

116. Présentation plus précise dans : SADDIER, Évelyne, Une seigneurie rurale..., op. cit., p. 27-32. 
frais. Les officiers pèsent donc modérément sur les finances seigneuriales : en moyenne, près d'un quart des dépenses totales leur est consacré ${ }^{117}$. Les gages, souvent modestes ${ }^{118}$, doivent pousser ces hommes à détenir d'autres biens ou revenus pour pouvoir subvenir à leurs besoins. Malheureusement, aucune trace n'apparaît dans les registres.

Intermédiaires entre les seigneurs et les tenanciers, quels liens les officiers entretiennent-ils avec le monde paysan? Les fonctions subalternes prise de ferme, levée des rentes - sont directement attribuées par le biais des enchères aux paysans, qui gardent un lien très fort avec leurs terres en continuant à les cultiver. Les fonctions plus spécialisées, procureurs, receveurs ou censiers, posent davantage question. Les noms insérés dans les comptes des Huguetières ne montrent pas de liens directs avec les tenanciers. Nous ne pouvons malheureusement pas connaître l'origine de ces officiers. Peut-être étaient-ils extérieurs aux territoires dont ils avaient la charge, de manière à pouvoir agir sans conflit d'intérêt. Les receveurs de La Blanchardais paraissent entretenir des liens plus étroits avec les tenanciers. Guillaume Leschardox, qui exerce entre 1457 (n. st.) et 1469 , mentionne à de nombreuses reprises d'autres paysans portant le nom de Leschardox; les registres restent malheureusement silencieux sur leurs éventuels liens de parenté. La faible dispersion du patrimoine des seigneurs de La Blanchardais, la longueur de l'exercice de Guillaume Leschardox, sa connaissance étroite du territoire sur lequel il officie sont autant d'éléments qui laissent penser que ce receveur était parent des tenanciers.

L'absence de registres de comptes pour les autres seigneuries oblige à se tourner vers d'autres sources. La confrontation des actes de la pratique montre que les moines de Buzay ont largement recours aux officiers. L'un des plus connus et des plus ambigus, par son action de rassemblement de terres et de constitution de métairies, demeure Jean Gauguet. Actif entre 1438 et $1473^{119}$, qualifié de " procureur " dans les aveux, il n'est pas un officier comme les autres : il agit en effet de façon indépendante et n'occupe pas de fonction précise au sein du monastère ${ }^{120}$. D'autres, les " procureurs généraux ", tel Olivier Brossaud, gèrent les affaires courantes de l'abbaye ${ }^{121}$, avec l'aide de notaires. D'autres encore, tels Pierre Chatau ou Pierre du Pouez, se chargent de défendre les intérêts des moines lors des procès ${ }^{122}$.

117. À la rubrique des gages d'officiers (par exemple, pour La Blanchardais, Arch. dép. de Loire-Atlantique, 1 E 223 [2], $\mathrm{f}^{\circ} 34 \mathrm{r}^{\circ}$ ) s'ajoutent ceux des receveurs, qui ne sont pas indiqués dans cette partie, mais aussi les versements effectués à d'autres personnes par mandements seigneuriaux, aujourd'hui perdus.

118. Le receveur Guillaume Leschardox reçoit ainsi chaque année (en 1467, 1468 et 1469) 10 livres pour exercer sa charge (Arch. dép. de Loire-Atlantique, 1 E $222(3), f^{\circ} 28 r^{\circ}$ ).

119. SARRAZIN, Jean-Luc, " Les activités d'un rassembleur de terres en Pays de Rais vers le milieu du Xve siècle ", Annales de Bretagne et des Pays de l'Ouest, t. 88, 1981, p. 136.

120. Ibidem, p. 142.

121. SARRAZIN, Jean-Luc, Recueil et catalogue..., op. cit., p. LXXXVII.

122. SARRAZIN, Jean-Luc, "Les activités d'un rassembleur... ", loc. cit, p. 142. 
Au total, les officiers sont plus nombreux car l'étendue des tâches et du patrimoine de Buzay l'exige.

\section{Les fermes}

Les fermes sont de plus en plus courantes et se diversifient tout au long du Xve siècle. L'exploitation des fours ou des moulins ${ }^{123}$, les ventes de bois, d'herbe ou de prés, la perception des terrages ${ }^{124}$ sont régulièrement accomplies sous cette forme. Le premier avantage des fermes réside en leur souplesse. Effectuées au gré des besoins, à n'importe quel moment, elles permettent d'adapter rapidement le prélèvement à la conjoncture en disposant immédiatement des revenus ${ }^{125}$. Elles reportent ensuite les risques et les aléas de la perception sur les fermiers. La multiplication des épisodes météorologiques violents ${ }^{126}$, accompagnés d'inondations ${ }^{127}$, conduisent les receveurs à y recourir pour contourner les difficultés et tenter de préserver les revenus du domaine. Les équilibres, très fragiles, sont parfois rompus par l'absence de preneurs ${ }^{128}$. Conjuguées aux effets extérieurs, ces pertes accentuent l'érosion des revenus seigneuriaux ${ }^{129}$.

Les fermes renvoient aussi aux équilibres sociaux. Elles permettent de saisir indirectement les évolutions de la communauté paysanne. Certains tenanciers, à l'instar des Chaléon (La Blanchardais), tirent parti des fermes pour s'élever progressivement dans la hiérarchie paysanne, en acquérant régulièrement celle du moulin de Vue ${ }^{130}$. L'affaiblissement de la valeur de la livre bretonne, consécutif aux mutations monétaires des années 1480, soutient les initiatives. L'ascension entraîne ensuite le reste du réseau familial, les héritiers emportant à leur tour les enchères. En continuant à cultiver leurs

123. RABOT, Brice, Les structures seigneuriales..., op. cit., p. 525-539.

124. Les receveurs des Huguetières les afferment régulièrement comme en 1406 pour les paroisses de Sainte-Croix-de-Machecoul et de Saint-Même-le-Tenu : Arch. dép. de LoireAtlantique, E 500 (2), $\mathrm{f}^{\circ} 14$.

125. Le preneur doit acquitter directement l'enchère avant de prendre la ferme.

126. Les comptes de La Blanchardais sont explicites avec les événements de 1457, 1458, 1462, 1467, 1468, 1472, 1473, 1474, 1475, 1484, 1485, 1487, 1488, 1489 et 1490 (Arch. dép. de Loire-Atlantique, 1 E 221 à 1 E 223).

127. Par exemple : "Pour l'an mil IIIIC LVIII et LIX ne se charge d'aucune levée de rox, pour ce que par le grant submergement des eaux, il n'a trouvé à qui en faire vente. " Arch. dép. de Loire-Atlantique, 1 E 222 (1), $\mathrm{f}^{\circ} 19 \mathrm{r}^{\circ}$.

128. "Et ne se charge aucun prouffit de fermes pour les levées desdiz prez à la Chevalière du Grant Faucheis, du Petit Faucheis, ne des deux hommées Tutan, pour les ans mil IIIIC LVIII et LXIX. Pour ce que par le le [sic] grant submergement des eaux qui, celx ans, comblèrent et submergèrent lesdiz prez, l'on ne peut recuillir ne fournir prouffit nulles, ne aucunes des levées dicelx. Et ne tourna l'en que aucune chose en voulist donné par ferme ne aultrement. " Arch. dép. de Loire-Atlantique, 1 E 222 (1), $\mathrm{f}^{\circ} 8 \mathrm{r}^{\circ}$.

129. Le produit des fermes est toujours inférieur à celui des "rentes certaines " pour La Blanchardais dans les décennies 1470-1480 (RАвот, Brice, Les structures seigneuriales..., op. cit., graphique $\mathrm{n}^{\circ} 2$, p. 106).

130. Perrot Chaléon acquiert la ferme en 1431 pour 65 sous; Jehan Chaléon l'emporte en 1459 et 1462 pour 50 sous, tandis qu'en 1471 celle-ci s'élève à 40 sous (RABOT, Brice, Les structures seigneuriales..., op. cit., tableau $\mathrm{n}^{\circ}$ 7, p. 235). 
terres, les preneurs disposent en outre d'une base foncière suffisamment solide pour dégager les revenus nécessaires à l'acquisition des fermes.

La multiplication des fermes dans la seconde moitié du Xve siècle pose enfin la question de la motivation des preneurs. Les fermes procurent des profits suffisants pour susciter l'intérêt des paysans ${ }^{131}$. Par leur nombre et leur récurrence, les fermes supposent aussi que les preneurs disposent de liquidités pour les acquérir. Une grande partie des tenanciers, si l'on s'intéresse aux noms transcrits dans les registres, se trouve insérée dans les structures du prélèvement avec les prises de ferme, ce qui contribue à renforcer la légitimité des droits perçus. Loin d'être passives, les communautés paysannes, même dispersées, trouvent dans les fermes des outils pour renforcer leur cohésion et pour pouvoir prendre part au prélèvement. Aucune ferme ne paraît d'ailleurs avoir fait l'objet de contestations si l'on suit les registres transmis.

La reconstruction agraire opérée à la fin du Moyen Âge s'accompagne d'autres modifications dans les structures du prélèvement. Les redevances partiaires connaissent, à la fin du $\mathrm{XV}^{\mathrm{e}}$ siècle, un regain d'intérêt en pays de Retz avec les progrès du métayage, surtout dans les terres étroitement gérées par les seigneurs ${ }^{132}$. Associés aux indications des registres des Huguetières, les fonds notariaux laissés par Sixte Nevouet, installé dans la région de Saint-Philbert-de-Grand-Lieu, livrent de précieux renseignements sur les étapes et les modalités de ces opérations. Ruinés par les crises successives, les tenanciers les plus fragiles cèdent peu à peu leurs terres aux moines de Buzay ou à des officiers agissant en leur nom ${ }^{133}$; les parcelles rachetées sont ensuite rassemblées pour constituer des exploitations plus grandes avant d'être confiées, pour une durée pouvant aller jusqu'à 9 ans ${ }^{134}$, à de nouveaux preneurs qui acceptent en échange le paiement de redevances partiaires ${ }^{135}$. Les seigneurs du pays de Retz, comme ceux du

131. La mise en pâture des bovins et la glandée des porcs dans la forêt de Machecoul représente, par exemple, jusqu'à 50 livres de ferme annuelle, ce qui signifie que les preneurs remportaient beaucoup plus d'argent pour pouvoir s'acquitter d'une telle somme (SADDIER, Évelyne, Une seigneurie rurale..., op. cit., tableaux p. 66 et 68).

132. La métairie est en Bretagne méridionale à la fin du Moyen Âge un mode de mise en valeur des réserves seigneuriales. Le paiement par le métayer de redevances partiaires renvoie à une forme de paiement d'un loyer.

133. SARRAZIN, Jean-Luc, "Les activités d'un rassembleur... ", art. cit., p. 147-151.

134. Les comptes des Huguetières présentent des situations analogues : "Item compte, ledit chastellain, de la métaerie de La Guillaudière affermée à Jehan Thébaud et Guillaume Gouillandeau pour neuff ans et neuff levées parfaictes et acomplies commancsans à la feste de la Pentecouste l'an mil IIII ${ }^{\mathrm{C}}$ IIII ${ }^{\mathrm{XX}}$ et unze et y finissans lesdiz neuff ans révoluz à la somme de seix escus d'or par chacun an oultre 25 soulz de rante deuz chacun an sur ladite métaerie et par chacun desdicts neuff ans, ouict biains, chacun garny de quatre beuffs et une charrette, et pour les gouverner et pour les négoces de ladicte chastellenie et rendront ladite métaerie ensemancée à la fin de ladite ferme et garnie d'angreix et partie des terres guerettées. "Arch. dép. de Loire-Atlantique, E 503 (1), f 19 ; cité dans SADDIER, Évelyne, Une seigneurie rurale..., op. cit., p. 59-60.

135. À La Petite Angle (Rouans), le bail, conclu pour une durée de 7 à 9 ans, répartit à part égale les récoltes entre les métayers et les moines de Buzay (Arch. dép. de LoireAtlantique, H 29). 
Poitou un demi-siècle plus tôt au sortir de la guerre de Cent Ans ${ }^{136}$, réorientent en profondeur les structures agraires en favorisant le métayage et les redevances partiaires. Les premières expériences menées dans les dernières années du $\mathrm{Xv}^{\mathrm{e}}$ siècle ne sont que les prémices. Elles demeurent encore limitées et ne s'expriment pleinement qu'au cours du siècle suivant.

\section{Les termes de paiement}

Les flux s'organisent par périodes, selon les termes fixés par la coutume et les usages, et varient selon les seigneuries et les produits. La première partie des registres, consacrée aux recettes et charges, permet de dégager les principaux termes de paiement. Le compte rendu en 1462 par Guillaume Lechardox, receveur de Vue (tableau 1 et figure 2), présente à ce sujet un exemple révélateur ${ }^{137}$ en distinguant les " rentes certaines " en deniers de celles en nature ${ }^{138}$, prélevées à d'autres dates.

Les "rentes certaines " en deniers s'organisent autour de deux termes principaux, à la fin août et à Noël, en lien avec le calendrier agricole et liturgique. À l'opposé, les "rentes certaines " en nature sont concentrées à Noël, après que les paysans aient pu vendre une partie de leurs récoltes. Les receveurs concentrent le paiement des principales rentes à la fin de l'année pour faciliter la perception et éviter les fraudes. Ainsi organisées, les rentes permettent de disposer de revenus réguliers pour faire face aux besoins des seigneurs, tout en permettant de vendre les surplus à l'extérieur. Les nouvelles baillées, effectuées au moment des successions ou des nouvelles concessions, peuvent le cas échéant modifier les termes ${ }^{139}$ et les adapter : elles servent de variables d'ajustement. Les comptes montrent que les receveurs, tout en respectant les coutumes, n'hésitent pas à y recourir, surtout à la fin du $\mathrm{XV}^{\mathrm{e}}$ siècle, au moment où les structures se réorganisent. Les campagnes s'animent donc, périodiquement, de flux reliant les tenures à la résidence seigneuriale, où sont centralisés les prélèvements.

\section{La mise en circulation des denrées agricoles}

Le choix du prélèvement en nature ou en argent, effectué par les seigneurs ou leurs agents, se traduit en un sens par la question suivante : qui va au marché vendre les produits, le paysan ou l'officier seigneurial?

136. MERLE, Louis, La métairie et l'évolution agraire de la Gâtine poitevine de la fin du Moyen Âge à la Révolution, Paris, SEVPEN, 1958, p. 49-62.

137. Ce registre est l'un des plus fournis et des plus détaillés de la série de La Blanchardais (Arch. Dép. de Loire-Atlantique, 1 E 222 [1]).

138. Elles occupent une grande partie de la première partie du compte, consacrée aux " recettes et charges" : Arch. Dép. de Loire-Atlantique, 1 E 222 (1), $\mathrm{f}^{\circ} 1 \mathrm{r}^{\circ}-3 \mathrm{v}^{\circ}, 15 \mathrm{r}^{\circ}-16 \mathrm{r}^{\circ}$. 139. Quelques exemples sont attestés dans les comptes de La Blanchardais : Arch. dép. de Loire-Atlantique, 1 E 221 (2), $\mathrm{f}^{\circ} 1 \mathrm{r}^{\circ}, 2 \mathrm{v}^{\circ} ; 1$ E $221(3), \mathrm{f}^{\circ} 1 \mathrm{v}^{\circ}, 2 \mathrm{r}^{\circ} ; 1$ E $221(4), \mathrm{f}^{\circ} 2 \mathrm{r}^{\circ}-\mathrm{v}^{\circ}, 13$ $\mathrm{r}^{\circ} ; 1$ E 222 (1), $\mathrm{f}^{\circ} 2 \mathrm{r}^{\circ}-\mathrm{v}^{\circ} ; 1$ E 222 (2), $\mathrm{f}^{\circ} 2 \mathrm{r}^{\circ}-\mathrm{v}^{\circ}, 4 \mathrm{v}^{\circ} ; 1$ E $222(3), \mathrm{f}^{\circ} 1 \mathrm{v}^{\circ}, 2 \mathrm{r}^{\circ}, 3 \mathrm{v}^{\circ} ; 1 \mathrm{E} 222$ (4), $\mathrm{f}^{\circ} 2 \mathrm{v}^{\circ}, 3 \mathrm{r}^{\circ} ; 1 \mathrm{E} 223(1), \mathrm{f}^{\circ} 6 \mathrm{r}^{\circ}, 7 \mathrm{v}^{\circ}, 10 \mathrm{v}^{\circ} ; 1 \mathrm{E} 223$ (2), $\mathrm{f}^{\circ} 6 \mathrm{r}^{\circ} ; 1 \mathrm{E} 223(3), \mathrm{f}^{\circ} 2 \mathrm{v}^{\circ} ; 1 \mathrm{E} 223$ (4), $3 \mathrm{v}^{\circ}, 4 \mathrm{r}^{\circ}, 6 \mathrm{v}^{\circ}, 7 \mathrm{v}^{\circ} ; 1 \mathrm{E} 223(5), \mathrm{f}^{\circ} 4 \mathrm{r}^{\circ} ; 1 \mathrm{E} 223(6), \mathrm{f}^{\circ} 3 \mathrm{r}^{\circ}, 6 \mathrm{r}^{\circ}$. 
Pour une nouvelle approche du prélèvement seigneurial

\section{Tableau 1 - Termes de paiement des "rentes certaines" selon le registre rendu en 1462}

\begin{tabular}{|c|c|c|c|c|c|}
\hline \multicolumn{3}{|c|}{ Vue } & \multicolumn{3}{|c|}{ Frossay } \\
\hline Terme & $\begin{array}{l}\text { Montant } \\
\text { (en livres } \\
\text { bretonnes) }\end{array}$ & $\begin{array}{l}\text { Part parmi } \\
\text { les recettes } \\
\text { totales en \% }\end{array}$ & Terme & $\begin{array}{l}\text { Montant } \\
\text { (en livres } \\
\text { bretonnes) }\end{array}$ & $\begin{array}{l}\text { Part parmi } \\
\text { les recettes } \\
\text { totales en \% }\end{array}$ \\
\hline Chandeleur & $11.5 \mathrm{~d}$. & 2,35 & $\begin{array}{l}\text { Pâques } \\
\text { fleuries } \\
\text { La date de } \\
\text { Pâques fleu- } \\
\text { ries est celle } \\
\text { du dimanche } \\
\text { des Rameaux; } \\
\text { elle change } \\
\text { donc chaque } \\
\text { année. }\end{array}$ & $10 \mathrm{~s}$. & 3,78 \\
\hline Mi-carême & $6 \mathrm{s.} 8 \mathrm{~d}$. & 0,77 & Madeleine & 1 1. $11 \mathrm{~s} .6 \mathrm{~d}$ & 11,9 \\
\hline Pâques & 41.17 s. $11 \mathrm{~d}$. & 11,27 & Septembre & $6 \mathrm{l} .10 \mathrm{~s} .9 \mathrm{~d}$ & 49,38 \\
\hline $\begin{array}{l}\text { Madeleine } \\
\text { (22 juillet) }\end{array}$ & 21.17 s. 5 d. & 6,61 & Saint-Viaud & 21. & 15,11 \\
\hline Mi-août & $7 \mathrm{l} .12$ s. 5 d. & 17,54 & \multirow{3}{*}{ Noël } & 2 1. 12 s. $6 \mathrm{~d}$ & 19,83 \\
\hline $\begin{array}{l}\text { St-Philbert } \\
\text { (20 août) }\end{array}$ & 19 s. 8 d. & 2,26 & & $\begin{array}{c}20 \text { boisseaux } \\
\text { de froment }\end{array}$ & 100 \\
\hline $\begin{array}{c}\text { Notre-Dame } \\
\text { de septembre }\end{array}$ & $41.8 \mathrm{~d}$. & 9,28 & & 9,5 chapons & 100 \\
\hline $\begin{array}{l}\text { Saint-Viaud } \\
\text { (16 octobre) }\end{array}$ & $3 \mathrm{l} .11 \mathrm{~s} .10 \mathrm{~d}$. & 8,27 & & & \\
\hline Toussaint & $10 \mathrm{l} .8 \mathrm{~s} .8 \mathrm{~d}$ & 24,02 & & & \\
\hline \multirow{6}{*}{ Noël } & $71.13 \mathrm{~s} .2 \mathrm{~d}$. & 17,63 & & & \\
\hline & $\begin{array}{l}24 \text { boisseau } \\
1 / 8 \text { e de fro- } \\
\text { ment }\end{array}$ & 100 & & & \\
\hline & $\begin{array}{c}18 \text { boisseaux } \\
\text { de seigle }\end{array}$ & 100 & & & \\
\hline & $\begin{array}{c}9 \text { truellées } \\
\text { d'avoine }\end{array}$ & 100 & & & \\
\hline & $\begin{array}{c}57 \text { chapons } \\
2 / 3\end{array}$ & 100 & & & \\
\hline & 6 poules & 100 & & & \\
\hline
\end{tabular}


Figure 2 - Répartition (en \%) des termes de paiement des " rentes certaines " en deniers (paroisses de Vue et Frossay)
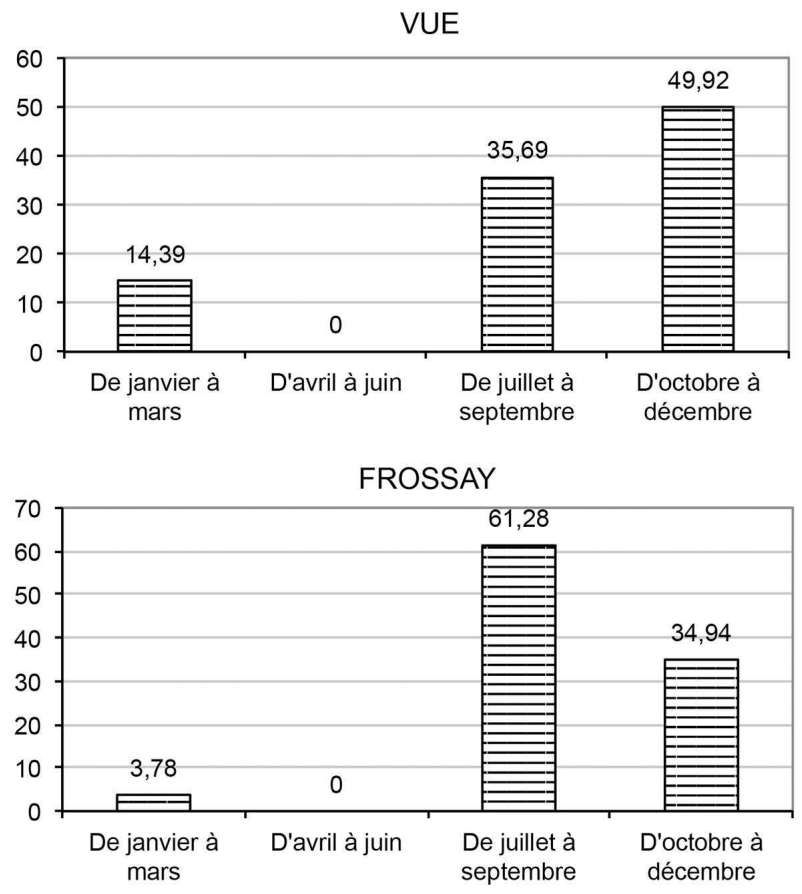

La redevance peut être prélevée au départ, au champ, comme pour les redevances partiaires, ou bien après la vente des produits par les paysans, qui n'ont d'ailleurs pas nécessairement besoin de se rendre dans les grandes villes pour cela. Les petits marchés ruraux, à peine visibles dans les sources ${ }^{140}$, révèlent en effet l'importance des relais locaux. La fréquence des redevances en argent oblige donc les tenanciers à écouler eux-mêmes leurs productions pour dégager l'argent nécessaire au paiement des rentes.

La deuxième partie consacrée aux mises et paiements apporte d'autres informations. Les rentes perçues sur les tenanciers sont, pour partie, réutilisées par les receveurs pour s'acquitter des rentes pesant sur les fiefs. Les receveurs de La Blanchardais versent ainsi chaque année quelques rentes en céréales et/ou en volailles aux sires de Retz ${ }^{141}$, aux seigneurs

140. Ils sont visibles à travers les droits de cohuage (Arch. Dép. De Loire-Atlantique, E $\left.522[1], \mathrm{f}^{\circ} \mathrm{f}^{\circ} 5 \mathrm{r}^{\circ}\right)$.

141. Arch. dép. de Loire-Alantique, 1 E 221 (3), f $\mathrm{f}^{\circ} 21 \mathrm{r}^{\circ}$; 1 E 222 (1), f $\mathrm{f}^{\circ} 25 \mathrm{r}^{\circ}$; 1 E 222 (2), $\mathrm{f}^{\circ} 24 \mathrm{v}^{\circ} ; 1 \mathrm{E} 222(4), \mathrm{f}^{\circ} 29 \mathrm{r}^{\circ} ; 1 \mathrm{E} 223(1), \mathrm{f}^{\circ} 25 \mathrm{v}^{\circ}$. 
de Bois-Rouaud (Frossay) ${ }^{142}$, de Vieillevigne ${ }^{143}$ ou aux moines de Buzay ${ }^{144}$. Les montants retranscrits demeurent modestes ${ }^{145}$. En se rendant à la cour du seigneur suzerain pour s'acquitter de la rente foncière, le receveur agit comme les tenanciers et reconnaît, au nom de son seigneur, l'autorité symbolique attachée au prélèvement. Cette opération est essentielle, car elle constitue l'une des bases des relations vassaliques à la fin du Moyen Âge. Les versements ont lieu après le paiement des tenanciers ${ }^{146}$, ce qui souligne une étroite articulation et nécessite une bonne gestion de la part des receveurs.

Les receveurs peuvent enfin revendre une partie des rentes en nature pour accroître les revenus seigneuriaux totaux. Les seuls exemples disponibles concernent la châtellenie des Huguetières : chaque année, les trois quarts des rentes en céréales sont ainsi vendus pour plusieurs dizaines de livres $^{147}$. Le patrimoine, diversifié, et le cumul des rentes en nature rendent possible ces échanges. À l'inverse, les seigneuries plus modestes ne disposent pas de surplus suffisants pour pouvoir les vendre à l'extérieur. Les receveurs de La Blanchardais, par exemple, ne font à aucun moment état de ventes de céréales, car les besoins domestiques des Blanchard accaparent le produit des rentes.

Le prélèvement ne se limite donc pas seulement à la bonne perception des redevances, il sert aussi à alimenter différents circuits, plus ou moins longs, dont les seigneurs tirent également profit.

Le croisement des approches permet en définitive de porter de nouveaux regards sur le prélèvement seigneurial. Divers dans ses formes, le prélèvement s'avère modéré pour les tenanciers, surtout pour les " rentes certaines ". L'approche économique révèle aussi des redevances fixes limitées, que ce soit sur les productions agricoles ou sur le travail paysan avec les corvées ou les bians. Les redevances partiaires présentent des traits bien différents. Les vignes, avec les complants, les terrages font certes

142. Arch. dép. de Loire-Atlantique, 1 E 222 (1), $\mathrm{f}^{\circ} 27 \mathrm{v}^{\circ} ; 1$ E 222 (2), $\mathrm{f}^{\circ} 27 \mathrm{v}^{\circ} ; 1$ E 222 (3), $\mathrm{f}^{\circ} 31 \mathrm{v}^{\circ} ; 1$ E 222 (4), $\mathrm{f}^{\circ} 34 \mathrm{r}^{\circ}$; 1 E 223 (1), $\mathrm{f}^{\circ} 29 \mathrm{v}^{\circ} ; 1$ E 223 (2), $\mathrm{f}^{\circ} 35 \mathrm{r}^{\circ} ; 1$ E 223 (5), $\mathrm{f}^{\circ} 25 \mathrm{r}^{\circ}$. 143. Arch. dép. de Loire-Atlantique, 1 E 222 (1), f $27 \mathrm{v}^{\circ} ; 1$ E 222 (2), $\mathrm{f}^{\circ} 27 \mathrm{v}^{\circ}-28 \mathrm{r}^{\circ}$; 1 E $222(3), f^{\circ} 31 v^{\circ}$.

144. Arch. dép. de Loire-Atlantique, 1 E 222 (1), $\mathrm{f}^{\circ} 27 \mathrm{v}^{\circ} ; 1$ E 222 (2), $\mathrm{f}^{\circ} 27 \mathrm{v}^{\circ}-28 \mathrm{r}^{\circ}$; $1 \mathrm{E} 222(3), \mathrm{f}^{\circ} 31 \mathrm{v}^{\circ}$.

145. Les sommes versées ne dépassent pas, à chaque fois, les dix sous et les quelques boisseaux de blé (jamais plus de quatre, bien que nous ne puissions pas convertir les mesures utilisées en notre système métrique).

146. Par exemple : «Et premier, à monseigneur de Rais, à cause de sa terre et seignorie de Rais, par la main de Jehan de La Roche, son chastelain dudit lieu, de rente deue à mondit seigneur, chacun an, au terme de meaougst, pour ledit terme mil IIIIC LVII, 25 soulz. Item, de rente li deue au terme de Toussains celui an mil IIIIC LVII, a poié 5 soulz. Item, à mondit seigneur de Rais, pour rente li deue au terme de Noël dit an mil IIIIC LVII, a poié 13 soulz 4 deniers, qu'est somme du tout desdictes rentes pour ledit an 43 soulz 4 deniers. " Arch. dép. de Loire-Atlantique, 1 E 222 (1), $\mathrm{f}^{\circ} 25 \mathrm{r}^{\circ}$.

147. SADDIER, Évelyne, Une seigneurie rurale..., op. cit., p. 100. 
l'objet de prélèvements moins nombreux mais beaucoup plus lucratifs pour les seigneurs, surtout lorsque d'autres rentes ne sont pas exigées. L'approche anthropologique conduit ensuite à réinterroger les rapports entre les seigneurs et les communautés paysannes. Les tenanciers, sur qui pèse l'essentiel des redevances, participent activement à la mise en œuvre du prélèvement, au travers des fermes ou des levées pour les rentes en deniers à " assembler en une seule main ". Ils ne sont plus seulement assujettis au paiement des redevances, mais en deviennent aussi des rouages essentiels. Le prélèvement semble dans ces conditions d'autant mieux accepté. L'approche géohistorique conduit enfin à réfléchir au poids décisif des influences extérieures dans l'organisation et la répartition spatiale des structures agraires. Les liens étroits avec les seigneurs poitevins, les échanges avec l'Anjou conduisent les seigneurs du pays de Retz à privilégier les redevances partiaires, comme leurs voisins, tout en s'appropriant, et en les adaptant, les systèmes d'exploitation rencontrés dans les provinces limitrophes.

\section{RÉSUMÉ}

L'historiographie récente invite à reconsidérer le thème du prélèvement seigneurial en croisant les approches et les démarches. Les modalités concrètes des levées, les rapports entretenus entre les officiers et les tenanciers ou encore la mise en circulation des denrées agricoles sont autant de pistes à explorer ou à revisiter. Le pays de Retz offre un cadre suffisamment ample et cohérent pour mener une telle étude. Occupant la majeure partie de la rive sud de la Loire, aux confins de l'Anjou et du Poitou, le pays de Retz est aussi l'un des territoires les mieux documentés pour la Bretagne méridionale à la fin du Moyen Âge. Sa position périphérique permet plus largement d'aborder la question du poids des influences extérieures dans l'orientation des structures agricoles et des types de redevances.

\section{ABSTRACT}

Recent historiography invites us to reconsider the theme of seigneurial levies by crossing different approaches and methodologies. The methods of payment, relationships between officers and farmers or, indeed, the distribution of agricultural products all deserve to be explored or revisited. The Retz region offers an ample and coherent enough framework within which to undertake such a study. Occupying the major part of the southern bank of the Loire, on the borders of Anjou and Poitou, Retz is also one of the best documented territories in Southern Brittany at the end of the Middle Ages. More broadly, its peripheral position allows us to examine the impact of the outside influences on the nature of agricultural structures and the types of duties. 